\title{
Low-energy effective Lagrangian from nonminimal supergravity with unified gauge symmetry
}

\author{
Yoshiharu Kawamura \\ Department of Physics, Shinshu University, Matsumoto, 390 Japan
}

(Received 1 August 1995)

\begin{abstract}
From general supergravity theory with unified gauge symmetry, we obtain the low-energy effective Lagrangian by taking the flat limit and integrating out the superheavy fields in a model-independent manner. The scalar potential possesses some excellent features. Some light fields classified by using a supersymmetric fermion mass, in general, would get intermediate masses at the tree level after the supersymmetry is broken. We show that the stability of the weak scale can be guaranteed under some conditions. There exist extra nonuniversal contributions to soft supersymmetry-breaking terms which can give an impact on phenomenological study.
\end{abstract}

PACS number(s): 04.65.+e, 12.10.-g, 12.60.Jv

\section{INTRODUCTION}

The minimal supersymmetric standard model (MSSM) is the most attractive candidate for a realistic theory beyond the standard model. The naturalness problem is elegantly solved by the introduction of supersymmetry (SUSY) [1]. SUSY requires new particles called "superpartners," whose masses are free parameters in the MSSM, ${ }^{1}$ but are estimated as at most of order $1 \mathrm{TeV}$ from the naturalness argument. The search for "superpartners" is one of the main purposes of the experimental projects using huge colliders, which have been planned now [2].

It is, however, believed that the MSSM is not the ultimate theory because there are many problems not solved by it. Here we pick out two problems. First, the MSSM lacks predictability since there are so many free parameters which can be fixed only by experiments for the present, such as gauge couplings, Yukawa couplings, and soft SUSY-breaking parameters. Second, the mechanism of SUSY breaking is unexplained. This problem is partly related to the first one since the pattern of soft SUSY-breaking terms depends on the SUSY-breaking mechanism.

It is expected that they are solved in a more fundamental theory. Supergravity (SUGRA) [3] is an attractive candidate. When we take SUGRA as an effective theory at the Planck scale $M_{\mathrm{Pl}}$, SUGRA offers the following interesting scenario [4]. SUSY is spontaneously or dynamically broken in the so-called hidden sector and the effect is transported to the observable sector by the gravitational interaction. In this scenario, the form of soft SUSY-breaking terms is determined by the structure of SUGRA.

The analyses based on the MSSM motivated by a minimal SUGRA are energetically investigated [5]. They are highly constrained by the assumption that the soft SUSY-breaking parameters are universal at the gravitational scale $M$ or a unification scale $M_{X}$. It is quite interesting because the theory has high predictabilities and is testable enough, but it is not necessarily realistic. Let us list the reasons. First the assumption of a universal scalar mass is motivated by the

\footnotetext{
${ }^{1}$ In this paper, we do not assume universality of the soft SUSYbreaking parameters from the beginning when we use the terminology "MSSM."
}

fact that flavor-changing neutral current $(\mathrm{FCNC})$ processes are suppressed experimentally [6]. However, we can relax this assumption since the suppression of FCNC processes due to SUSY particle loops requires only degeneracy among squarks with the same flavor. Second there is no strong reason that the realistic SUGRA takes the minimal structure. In fact, the effective SUGRA's derived from superstring theories (SST's) have, in general, nonminimal structures and they can lead to effective theories with nonuniversal soft parameters [7]. And it was pointed out that higher order corrections generally destroy the minimal form of the Kähler potential [8]. Last the effects of SUSY grand unified theory (GUT) were little considered although SUSY GUT [9] has been hopeful as a realistic theory. The unification dogma [10] has merit in that the number of independent parameters is reduced due to a large gauge symmetry. Further SUSY SU(5) GUT is supported by the experiments [11] at the CERN $e^{+} e^{-}$collider LEP and predicts a long lifetime of nucleons consistent with the present data [12]. Even if we take a minimal SUGRA as a starting point, the radiative correction from $M$ to $M_{X}$ changes the universal form of SUSY-breaking terms into a nonuniversal one. These renormalization effects were discussed [13], but we need to consider effects on the symmetry breaking further. It is shown that new contributions to SUSY-breaking terms can appear at the tree level after integrating out superheavy fields [14]. Analyses including the effects were started recently [15].

Now we should stress the importance of studying the soft SUSY-breaking terms. The reason is that they can be a powerful probe to SUSY GUT, SUGRA, and/or SST since the weak scale SUSY spectrum can directly reflect the physics at very high energies. For example, we can check the GUT scenario experimentally by measuring the gaugino masses [16]. Also, the scalar mass spectrum has certain "sum rules" specific to symmetry-breaking patterns [17]. Therefore, precision measurements of the SUSY spectrum are very important. And it is a meaningful subject to obtain the low-energy theory in a more general framework and to grasp the peculiarities concerning the SUSY-breaking terms in advance.

In this paper, we derive the low-energy effective theory from nonminimal SUGRA with unified gauge symmetry. We write down the theory in terms of SUGRA in order to get information on the structure of SUGRA directly. The starting SUGRA has a more general structure than those considered before $[18,19,14]$; i.e., the Kähler potential is nonminimal 
and we do not impose a hidden ansatz on the superpotential. Here the hidden ansatz means that the superpotential is separate from the hidden sector to the visible one. Then dangerous terms, which destabilize the gauge hierarchy, generally appear at the tree level. We discuss conditions that the hierarchy is preserved, and take the flat limit and integrate out superheavy fields without identifying $M_{X}$ with $M$. We find various contributions to the SUSY-breaking terms. It is shown that our result reduces to that obtained in Ref. [14] in the limit $M_{X} / M \rightarrow 0$ when we take a certain type of total Kähler potential.

The paper is organized as follows. In Sec. II, we first review the low-energy effective Lagrangians from SUGRA following the historical development. We derive the lowenergy effective scalar potential starting from SUGRA with a general total Kähler potential and unified gauge symmetry in Sec. III. In Sec. IV, we discuss $D$-term contributions to scalar masses and make clear the relation between our result and that in Ref. [14]. Section V is devoted to conclusions.

\section{HISTORICAL BACKGROUND}

\section{A. Scalar sector in SUGRA}

We begin by reviewing the scalar sector in SUGRA [3]. It is specified by two functions, the total Kähler potential $G\left(z, z^{*}\right)$ and the gauge kinetic function $f_{\alpha \beta}(z)$ with $\alpha, \beta$ being indices of the adjoint representation of the gauge group. The former is a sum of the Kähler potential $K$ and (the logarithm of) the superpotential $W_{\mathrm{SG}}$ such as

$$
G\left(z, z^{*}\right)=K\left(z, z^{*}\right)+M^{2} \ln \left|W_{\mathrm{SG}}(z) / M^{3}\right|^{2} .
$$

We have denoted the scalar components of chiral multiplets by $z^{I}$ and their complex conjugates by $z_{J}^{*}$. The scalar potential is given by

$$
V=M^{2} e^{G / M^{2}} U+\frac{1}{2}\left(\operatorname{Re} f^{-1}\right)_{\alpha \beta} D^{\alpha} D^{\beta},
$$

where

$$
\begin{gathered}
U=G^{I}\left(K^{-1}\right)_{I}^{J} G_{J}-3 M^{2}, \\
D^{\alpha}=G_{I}\left(T^{\alpha} z\right)^{I}=\left(z^{\dagger} T^{\alpha}\right)_{J} G^{J} .
\end{gathered}
$$

Here $G_{I}=\partial G / \partial z^{I}, G^{J}=\partial G / \partial z_{J}^{*}$, etc., and $T^{\alpha}$ are gauge transformation generators. Also $\left(\operatorname{Re} f^{-1}\right)_{\alpha \beta}$ and $\left(K^{-1}\right)_{I}^{J}$ are the inverse matrices of $\operatorname{Re} f_{\alpha \beta}$ and $K_{I}^{J}$ respectively, and summation over $\alpha, \ldots$ and $I, \ldots$ is understood.

Let us next summarize our requirements on the SUSY breaking. The gravitino mass $m_{3 / 2}$ is given by

$$
m_{3 / 2}=\left\langle e^{K / 2 M^{2}} \frac{W_{\mathrm{SG}}}{M^{2}}\right\rangle,
$$

where angular brackets denote the vacuum expectation value (VEV) of the quantity. We identify the gravitino mass with the weak scale. The $F$ auxiliary fields of the chiral multiplets are defined as $F^{I} \equiv M e^{G / 2 M^{2}}\left(K^{-1}\right)_{J}^{I} G^{J}$ and we require that those VEV's should satisfy $\left\langle F^{I}\right\rangle \leqslant O\left(m_{3 / 2} M\right)$. It follows that $\left\langle G_{I}\right\rangle,\left\langle G^{J}\right\rangle \leqslant O(M)$ and $\langle U\rangle \leqslant O\left(M^{2}\right)$. Note that we allow the nonzero vacuum energy $\langle V\rangle$ of order $m_{3 / 2}^{2} M^{2}$ at this level, which could be canceled by quantum corrections. We can show that the VEV's of the $D$ auxiliary fields become very small, $\left\langle D^{\alpha}\right\rangle \leqslant O\left(m_{3 / 2}^{2}\right)$, as will be shown in Appendix A. We shall call the fields which induce the SUSY breaking "hidden fields" and denote those scalar components and $F$ components as $\tilde{z}^{i}$ and $\tilde{F}^{i}$, respectively. We require that those VEV's should satisfy $\left\langle\tilde{z}^{i}\right\rangle=O(M)$ and $\left\langle\tilde{F}^{i}\right\rangle=O\left(m_{3 / 2} M\right)$. We shall call the rest "observable fields" and denote the scalar components as $z^{\kappa}$.

\section{B. Effective theories from minimal SUGRA}

Minimal SUGRA has a canonical Kähler potential $K=\left|z^{\kappa}\right|^{2}+\left|\tilde{z}^{i}\right|^{2}$. We take the hidden ansatz for the superpotential as $W_{\mathrm{SG}}=W(z)+\tilde{W}(\tilde{z})$. After we take the flat limit, i.e., $M \rightarrow \infty$ but $m_{3 / 2}$ kept finite, we obtain a scalar potential such as [4]

$$
V=V_{\mathrm{SUSY}}+V_{\mathrm{soft}}
$$

$$
\begin{gathered}
V_{\mathrm{SUSY}}=\left|\frac{\partial \hat{W}}{\partial z^{\kappa}}\right|^{2}+\frac{1}{2} g_{\alpha}^{2}\left[z_{\kappa}^{*}\left(T^{\alpha}\right)_{\lambda}^{\kappa} z^{\lambda}\right]^{2}, \\
V_{\mathrm{soft}}=A \hat{W}+B z^{\kappa} \frac{\partial \hat{W}}{\partial z^{\kappa}}+\text { H.c. }+|B|^{2} z_{\kappa}^{*} z^{\kappa},
\end{gathered}
$$

where $\hat{W}$ is defined as $\hat{W} \equiv\left\langle\exp \left(K / 2 M^{2}\right)\right\rangle W . V_{\text {SUSY }}$ stands for the supersymmetric part, while $V_{\text {soft }}$ contains the soft SUSY-breaking terms. The parameters $A$ and $B$ are written as

$$
\begin{gathered}
A=\frac{\left\langle\tilde{F}^{i}\right\rangle\left\langle K_{i}\right\rangle}{M^{2}}-3 m_{3 / 2}^{*}, \\
B=m_{3 / 2}^{*} .
\end{gathered}
$$

This form of SUSY-breaking terms is referred to as "universal.",

The low-energy scalar potential is obtained from minimal SUGRA with a unified gauge symmetry by taking the flat limit and integrating out superheavy fields simultaneously on the postulation that the unification scale $M_{X}$ is identified with $M[18]$ :

$$
\begin{gathered}
V^{\mathrm{eff}}=V_{\mathrm{SUSY}}^{\mathrm{eff}}+V_{\mathrm{soft}}^{\mathrm{eff}}, \\
V_{\mathrm{SUSY}}^{\mathrm{eff}}=\mid \frac{\left.\partial \hat{W}_{\mathrm{eff}}\right|^{2}}{\partial z^{k}}+\frac{1}{2} g_{a}^{2}\left[z_{k}^{*}\left(T^{a}\right)_{l}^{k} z^{l}\right]^{2}, \\
V_{\mathrm{soft}}^{\mathrm{eff}}=A \hat{W}_{\mathrm{eff}}+B z^{k} \frac{\partial \hat{W}_{\mathrm{eff}}}{\partial z^{k}}+\text { H.c. }+|B|^{2} z_{k}^{*} z^{k}+\Delta V, \\
\Delta V \equiv-3 A \hat{W}_{\mathrm{eff}}+A z^{k} \frac{\partial \hat{W}_{\mathrm{eff}}}{\partial z^{k}}+\text { H.c. }
\end{gathered}
$$


where $z^{k}$ are the light scalar fields, ${ }^{2} a$ is the index of generators of unbroken gauge group, and $\hat{W}_{\text {eff }}$ is the superpotential $\hat{W}$ with the extremum values for superheavy fields plugged in. The scalar mass terms are still universal with the same mass $B .^{3}$

The universal structure of the low-energy Lagrangian led to a number of strong conclusions, like the natural absence of flavor-changing neutral currents [6] or the radiative breaking scenario due to the heavy top quark [20]. Because of these successes, the phenomenological analysis has become popular based on SUSY models with universal soft SUSYbreaking terms [5]. However, it becomes increasingly apparent that SUGRA may not have the minimal form, and it is important to study the consequences on the low-energy effective Lagrangian.

\section{Effective theories from nonminimal SUGRA}

The scalar potential is also obtained from nonminimal SUGRA [21]:

$$
\begin{gathered}
V^{(\text {non })}=V_{\mathrm{SUSY}}^{(\text {non })}+V_{\mathrm{soft}}^{(\text {non })} \\
V_{\mathrm{SUSY}}^{(\text {non })}=\left|\frac{\partial \hat{\mathscr{W}}}{\partial z^{\kappa}}\right|^{2}+\frac{1}{2} g_{\alpha}^{2}\left[z_{\kappa}^{*}\left(T^{\alpha}\right)_{\lambda}^{\kappa} z^{\lambda}\right]^{2} \\
V_{\mathrm{soft}}^{(\mathrm{non})}=A \hat{\mathscr{W}}+B^{\kappa}(z) \frac{\partial \hat{\mathscr{W}}}{\partial z^{\kappa}}+\text { H.c. }+B^{\kappa}(z) B_{\kappa}(z)+C\left(z, z^{*}\right),
\end{gathered}
$$

where

$$
\begin{aligned}
& B^{\kappa}(z)=m_{3 / 2}^{*} z^{\kappa}-K_{j}^{\kappa}\left\langle\tilde{F}^{j}\right\rangle, \\
C\left(z, z^{*}\right)= & -\left\langle\tilde{F}^{i}\right\rangle \delta^{2} K_{i}^{j}\left\langle\tilde{F}_{j}^{*}\right\rangle \\
& +\left\{\frac{1}{M^{2}}\left\langle\tilde{F}^{i}\right\rangle\left\langle K_{i}^{j}\right\rangle\left\langle\tilde{F}_{j}^{*}\right\rangle-3\left|m_{3 / 2}\right|^{2}\right\} \delta^{2} K \\
& +m_{3 / 2}\left\langle\tilde{F}^{i}\right\rangle \delta^{2} K_{i}+\text { H.c. } \\
& -A\left\{m_{3 / 2} H(z)-\left\langle\tilde{F}_{i}^{*}\right\rangle H^{i}(z)\right\}+\text { H.c. },
\end{aligned}
$$

in the case that we take the hidden ansatz. Here $\hat{\mathscr{W}}$ is defined as

$$
\hat{\mathscr{W}} \equiv \hat{W}+m_{3 / 2} H(z)-\left\langle\tilde{F}_{i}^{*}\right\rangle H^{i}(z)
$$

where $H(z)$ is the holomorphic part of $z^{\kappa}$ in $K$. And $\delta^{2} K$, $\delta^{2} K_{i}$, and $\delta^{2} K_{i}^{j}$ are the quantities of order $m_{3 / 2}^{2}, m_{3 / 2}^{2} / M$, and $m_{3 / 2}^{2} / M^{2}$ in $K, K_{i}$, and $K_{i}^{j}$, respectively. Note that the

\footnotetext{
${ }^{2}$ Hall et al. assumed that the supersymmetric masses of light fields from the superpotential are zero. It is straightforward to generalize their analysis into the case that the light fields have nonzero but $O\left(m_{3 / 2}\right)$ masses.

${ }^{3}$ Throughout this subsection, it is assumed that the vacuum energy $\langle V\rangle$ vanishes. In the presence of the vacuum energy, the value of scalar mass $|B|^{2}$ is replaced by $|B|^{2}+\langle V\rangle / M^{2}$.
}

SUSY-breaking terms show a nonuniversal form. As an excellent feature, the $\mu$ parameter of order $m_{3 / 2}$ originates in the second and third terms in Eq. (20) [22].

When the hidden ansatz is taken off, the following extra terms should be added:

$$
\frac{\partial \hat{\mathscr{W}}^{*}}{\partial \tilde{z}_{i}^{*}}\left\langle\left(K^{-1}\right)_{i}^{j}\right\rangle \frac{\partial \hat{\mathscr{W}}}{\partial \tilde{z}^{j}}+\Delta C\left(z, z^{*}\right)+\left\langle\tilde{F}^{i}\right\rangle \frac{\partial \hat{\mathscr{W}}}{\partial \tilde{z}^{i}}+\text { H.c. }
$$

where $\Delta C\left(z, z^{*}\right)$ is a bilinear polynomial of $z$ and $z^{*}$. The magnitude of the third term and its Hermitian conjugate can be of order $m_{3 / 2}^{3} M$, and so a large mixing mass of Higgs doublets can be introduced. Hence we need to impose the condition

$$
\left\langle\tilde{F}^{i}\right\rangle \frac{\partial \hat{\mathscr{W}}}{\partial \tilde{z}^{i}}=O\left(m_{3 / 2}^{4}\right)
$$

to guarantee the stability of the weak scale.

Effective theories based on nonminimal SUGRA with unified gauge symmetry also have been studied in the literature, but a complete analysis has not been carried out yet. For example, Hall et al. showed that the universality of scalar masses is preserved in SUGRA whose Kähler potential has $\mathrm{U}(n)$ symmetry among the $n$ chiral fields [18]. Drees studied the low-energy theory based on SUGRA with a noncanonical kinetic function parametrized by one chiral field which triggers the SUSY breaking [19].

As a recent development, the low-energy effective theory has been derived from SUSY GUT with a certain type of nonuniversal soft SUSY-breaking terms, which can be derived from a certain type of nonminimal SUGRA with unified gauge symmetry and the hidden ansatz by taking the flat limit first [14],

$$
\begin{aligned}
& V^{\mathrm{eff}(\text { non })}=V_{\mathrm{SUSY}}^{\mathrm{eff}(\text { non })}+V_{\mathrm{soft}}^{\mathrm{eff}(\mathrm{non})}, \\
& V_{\mathrm{SUSY}}^{\mathrm{eff(non})}=\left|\frac{\partial \hat{\mathscr{W}}_{\mathrm{eff}}}{\partial z^{k}}\right|^{2}+\frac{1}{2} g_{a}^{2}\left[z_{k}^{*}\left(T^{a}\right){ }_{l}^{k} z^{l}\right]^{2}, \\
& V_{\text {soft }}^{\mathrm{eff}(\mathrm{non})}= A \hat{\mathscr{W}}_{\mathrm{eff}}+B^{k}(z)_{\mathrm{eff}} \frac{\partial \hat{\mathscr{W}}_{\mathrm{eff}}}{\partial z^{k}}+\text { H.c. } \\
&+B^{k}(z)_{\mathrm{eff}} B_{k}(z)_{\mathrm{eff}}+C\left(z, z^{*}\right)_{\mathrm{eff}} \\
&+\Delta V^{(\text {non })},
\end{aligned}
$$

where $\hat{\mathscr{W}}_{\text {eff }}, B^{k}(z)_{\text {eff }}$, and $C\left(z, z^{*}\right)_{\text {eff }}$ are $\hat{\mathscr{W}}, B^{k}(z)$, and $C\left(z, z^{*}\right)$ with the extremum values for superheavy fields plugged in, and $\Delta V^{(\text {non })}$ is a sum of extra contributions specific to the extra gauge symmetry breaking. The most important one is a $D$-term contribution to the scalar masses. ${ }^{4}$ In the absence of a Fayet-Iliopoulos $D$ term, sizable $D$-term contributions can appear under the following conditions. (1) SUSY

\footnotetext{
${ }^{4}$ Historically, it was demonstrated that the $D$-term contribution occurs when the gauge symmetry is broken at an intermediate scale due to the nonuniversal soft scalar masses in Ref. [23] and its existence in a more general situation was suggested in Ref. [24].
} 
GUT has nonuniversal soft SUSY-breaking terms. (2) The rank of the gauge group is reduced by the gauge symmetry breaking. As the other feature, the gauge hierarchy achieved by a fine-tuning in the superpotential is preserved for SUSYGUT models derived from the SUGRA with the hidden ansatz and no light observable singlets after the SUSY breaking.

In the next section, we derive the low-energy scalar potential from a more general SUGRA and write it down in terms of SUGRA.

\section{DERIVATION OF THE EFFECTIVE LAGRANGIAN}

\section{A. Basic assumptions}

We shall first list our basic assumptions based on the hidden sector SUSY-breaking scenario.

(1) At the gravitational scale $M$, the theory is described effectively as nonminimal SUGRA with a certain unified gauge symmetry whose Kähler potential and superpotential are given as

$$
K=\tilde{K}\left(\tilde{z}, \tilde{z}^{*}\right)+\Lambda\left(z, z^{*} ; \tilde{z}, \tilde{z}^{*}\right)+H\left(z ; \tilde{z}, \tilde{z}^{*}\right)+\text { H.c. }
$$

and

$$
\begin{gathered}
W_{\mathrm{SG}}=\tilde{W}(\tilde{z})+W(z, \tilde{z}), \\
W(z, \tilde{z}) \equiv \frac{1}{2} m_{\kappa \lambda}(\tilde{z}) z^{\kappa} z^{\lambda}+\frac{1}{3 !} f_{\kappa \lambda \mu}(\tilde{z}) z^{\kappa} z^{\lambda} z^{\mu}+\cdots,
\end{gathered}
$$

respectively. Here the ellipsis stands for terms of higher orders in $z$. The gauge group is not necessarily grand unified into a simple group. The theory has no Fayet-Iliopoulos U(1) $D$ term for simplicity. ${ }^{5}$

(2) SUSY is spontaneously broken by the $F$-term condensation in the hidden sector. The hidden fields are gauge singlets and they have the VEV's of $O(M)$. The magnitudes of $W_{\mathrm{SG}}$ and $F$ component $\tilde{F}^{i}$ of $\tilde{z}^{i}$ are $O\left(m_{3 / 2} M^{2}\right)$ and $O\left(m_{3 / 2} M\right)$, respectively. The derivatives of the Kähler potential with respect to $z$ and $z^{*}$ are at most of order unity (in units where $M$ is taken to be unity), namely, $\left\langle K_{I_{1} \ldots}^{J_{1} \cdots}\right\rangle \leqslant O(1)$. This will be justified if Planck scale physics plays an essential role in the SUSY breaking. ${ }^{6}$

(3) The gravitino mass is of order of the weak scale. ${ }^{7}$

(4) The unified gauge symmetry is broken at a scale $M_{X}$ independent of the SUSY breaking. Our vacuum solution

\footnotetext{
${ }^{5}$ The extension of the theory with the Fayet-Iliopoulos $D$ term [25] is straightforward. We discuss it in Appendix B.

${ }^{6}$ Our discussion is also applicable to the case of SUSY breaking by gaugino condensation if the freedoms are effectively replaced by some scalar multiplets whose VEV's are of order $M$ as the models derived from SST.

${ }^{7}$ This assumption is a little too strong since we only need to require that the soft SUSY-breaking masses be of order of the weak scale. In fact, there is quite an interesting scenario [26] that the gravitino mass is decoupled to the soft parameters and the magnitude of SUSY breaking is determined by the gaugino masses.
}

$\left\langle z^{\kappa}\right\rangle$ is near to that $z_{0}^{\kappa}$ in the SUSY limit, i.e., $\left\langle z^{\kappa}\right\rangle=z_{0}^{\kappa}+O\left(m_{3 / 2}\right) .{ }^{8}$ Some observable scalar fields have the VEV's of $O\left(M_{X}\right)$.

(5) All the particles can be classified as heavy [with mass $O\left(M_{X}\right)$ ] or light [with mass $O\left(m_{3 / 2}\right)$ ]. There are no light singlet fields which induce a large tadpole contribution to Higgs masses by coupling to Higgs doublets renormalizably in the superpotential. For simplicity, the light observable fields are gauge nonsinglets and have fluctuations only of $O\left(m_{3 / 2}\right)$. The fields classification is stable irrespective of the SUSY breaking; i.e., the weak scale does not destabilize due to the SUSY breaking. The $D$-term contribution to the scalar masses for the light fields is not larger than the $F$ term one.

(6) We study the case that the equalities hold for the estimations of the scalar masses for simplicity.

\section{B. Vacuum solutions}

The scalar potential is given as

$$
\begin{gathered}
V=V^{(F)}+V^{(D)}, \\
V^{(F)} \equiv M^{2} \exp \left(G / M^{2}\right)\left[G^{I}\left(G^{-1}\right)_{I}^{J} G_{J}-3 M^{2}\right],
\end{gathered}
$$

$$
V^{(D)} \equiv \frac{1}{2}\left(\operatorname{Re} f^{-1}\right)_{\alpha \beta} D^{\alpha} D^{\beta}
$$

The index $I, J, \ldots$ runs all scalar species, $i, j, \ldots$ runs the hidden fields, and $\kappa, \lambda, \ldots$ runs the observable fields. The $D^{\alpha}$ 's are deformed as

$$
D^{\alpha}=K_{\kappa}\left(T^{\alpha} z\right)^{\kappa}=\left(z^{\dagger} T^{\alpha}\right)_{\kappa} K^{\kappa}
$$

from the gauge invariance of the superpotential. ${ }^{9}$

The vacuum solution $\left\langle z^{I}\right\rangle$ is determined by solving the stationary conditions $\partial V / \partial z^{I}=0$. The conditions that the SUSY not be spontaneously broken in the observable sector are simply expressed as $\partial W / \partial z^{\kappa}=0$ and $D^{\alpha}=0$. We denote the solutions of the above conditions as $z^{\kappa}=z_{0}^{\kappa}$.

The supersymmetric fermion mass $\mu_{I J}$ is given as

$$
\mu_{I J}=\left\langle M e^{G / 2 M^{2}}\left(G_{I J}+\frac{G_{I} G_{J}}{M^{2}}-G_{I^{\prime}}\left(G^{-1}\right)_{J^{\prime}}^{I^{\prime}} G_{I J}^{J^{\prime}}\right)\right\rangle .
$$

We take a basis of $z^{I}$ to diagonalize the SUSY fermion mass matrix $\mu_{I J}$. Then we assume that the scalar fields are classified either as "heavy" complex fields $z^{K}, z^{L}, \ldots$, "light" fields $z^{k}, z^{l}, \ldots, z^{i}, z^{j}, \ldots$ such as $\mu_{K L}=O\left(M_{X}\right)$, $\mu_{k l}=O\left(m_{3 / 2}\right), \mu_{i j}=O\left(m_{3 / 2}\right)$, or Nambu-Goldstone fields $z^{A}, z^{B}, \ldots$ (which will be discussed just below). The observable fields $z^{\kappa}$ consist of $z^{K}, z^{k}$, and $z^{A}$. It is shown that the hidden fields $z^{i}$ belong to the light sector in Appendix A.

The mass matrix of the gauge bosons $\left(M_{V}^{2}\right)^{\alpha \beta}$ is given as

\footnotetext{
${ }^{8} \mathrm{We}$ can show that there exists at least such a vacuum solution in the case that the scalar potential has no flat directions in the SUSY limit.

${ }^{9}$ When the superpotential is not gauge invariant under a U(1) transformation, the Fayet-Iliopoulos $D$ term exists [27].
} 


$$
\left(M_{V}^{2}\right)^{\alpha \beta}=2\left\langle\left(z^{\dagger} T^{\beta}\right){ }_{\kappa} K_{\lambda}^{\kappa}\left(T^{\alpha} z\right)^{\lambda}\right\rangle
$$

up to the normalization due to the gauge coupling constants, and it can be diagonalized so that the gauge generators are classified into "heavy" (those broken at $\left.M_{X}\right) T^{A}, T^{B}, \ldots$ and "light" (which remain unbroken above $m_{3 / 2}$ ) $T^{a}, T^{b}, \ldots$. For the heavy generators, the fields $\left\langle\left(T^{A} z\right)^{\kappa}\right\rangle$ correspond to the directions of the Nambu-Goldstone fields in the field space, which span a vector space with the same dimension as the number of heavy generators. We can take a basis of the Nambu-Goldstone multiplets, $z^{A}, z^{B}, \ldots$, so that

$$
\sqrt{2}\left\langle\left(T^{A} z\right)^{B}\right\rangle=M_{V}^{A B}
$$

Here the Nambu-Goldstone fields are taken to be orthogonal to the heavy and light fields such as $\left\langle\left(T^{A} z\right)^{K}\right\rangle=0$ and $\left\langle\left(T^{A} z\right)^{k}\right\rangle=0$. To be more precise, the imaginary parts of the $z^{A}$, s are the true Nambu-Goldstone bosons which are absorbed into the gauge bosons, and the real parts acquire the same mass of order $M_{X}$ as that of the gauge bosons from the $D$ term $V^{(D)}$ in the SUSY limit. Hence the Nambu-Goldstone multiplets belong to the heavy sector.

Let us give the procedure to obtain the low-energy effective theory.

(1) We calculate the VEV's of the derivatives of the potential and we write down the potential as

$$
V=\frac{1}{2}\left\langle V_{I J}\right\rangle \Delta z^{I} \Delta z^{J}+\cdots,
$$

where the scalar fields $z^{I}$ 's are expanded as $z^{I}=\left\langle z^{I}\right\rangle+\Delta z^{I}$ around the vacuum $\left\langle z^{I}\right\rangle$.

(2) When there exists a mass mixing between the heavy and light sectors, we need to diagonalize them to identify the light and heavy fields correctly.

(3) We solve the stationary conditions of the potential for the heavy scalar fields while keeping the light scalar fields arbitrary and then integrate out the heavy fields by inserting the solutions of the stationary conditions into the potential. We take the flat limit simultaneously.

\section{Derivatives of $K$ and $W$}

It is convenient to write both the Kähler potential $K$ and the superpotential $W_{\mathrm{SG}}$ in terms of the variations $\Delta z^{I}$ and $\Delta z_{J}^{*}$ as

$$
\begin{aligned}
K= & \langle K\rangle+\left\langle K_{I}\right\rangle \Delta z^{I}+\left\langle K^{J}\right\rangle \Delta z_{J}^{*}+\left\langle K_{I}^{J}\right\rangle \Delta z^{I} \Delta z_{J}^{*} \\
& +\frac{1}{2}\left\langle K_{I J}\right\rangle \Delta z^{I} \Delta z^{J}+\frac{1}{2}\left\langle K^{I J}\right\rangle \Delta z_{I}^{*} \Delta z_{J}^{*}+\cdots
\end{aligned}
$$

and

$$
\begin{aligned}
W_{\mathrm{SG}}= & \left\langle W_{\mathrm{SG}}\right\rangle+\left\langle W_{\mathrm{SG} I}\right\rangle \Delta z^{I}+\frac{1}{2}\left\langle W_{\mathrm{SG} I J}\right\rangle \Delta z^{I} \Delta z^{J} \\
& +\frac{1}{3 !}\left\langle W_{\mathrm{SGIJJ}}\right\rangle \Delta z^{I} \Delta z^{J} \Delta z^{J^{\prime}}+\cdots,
\end{aligned}
$$

By using the expansions (37) and (38), we find the estimations

$$
\begin{gathered}
\left\langle G_{i}\right\rangle=O(M), \quad\left\langle G_{K}\right\rangle \leqslant O\left(M_{X}\right), \\
\left\langle G_{A}\right\rangle \leqslant O\left(m_{3 / 2}^{2} / M_{X}\right), \quad\left\langle G_{k}\right\rangle=0, \\
\left\langle G_{i}^{j}\right\rangle \lesssim 1, \quad\left\langle G_{\kappa}^{\lambda}\right\rangle \lesssim 1, \quad\left\langle G_{K}^{j}\right\rangle \leqslant O\left(M_{X} / M\right), \\
\left\langle G_{A}^{j}\right\rangle \leqslant O\left(M_{X} / M\right), \quad\left\langle G_{k}^{j}\right\rangle=0,
\end{gathered}
$$

$$
\begin{gathered}
\left\langle G_{i j}\right\rangle \lesssim 1, \quad\left\langle G_{K L}\right\rangle \leqslant O\left(M_{K L} / m_{3 / 2}\right), \quad\left\langle G_{K j}\right\rangle \leqslant O\left(M_{X} / M\right), \\
\left\langle G_{A j}\right\rangle \leqslant O\left(M_{X} / M\right), \quad\left\langle G_{k j}\right\rangle=0 \\
\left\langle G_{\kappa B}\right\rangle \lesssim 1, \quad\left\langle G_{\kappa l}\right\rangle \lesssim 1
\end{gathered}
$$

where $M_{K L}$ is the SUSY fermion mass coming from the superpotential. Here we used the assumption that our vacuum solution is near to that in the SUSY limit and a perturbative argument to derive the second relation in (39). And we used the relations (35) and $\left\langle D^{\alpha}\right\rangle \leqslant O\left(m_{3 / 2}^{2}\right)$ to derive the third relation in (39).

By using the equality from the gauge invariance (A3), we derive the relations

$$
\begin{gathered}
\left\langle G_{A k l}\right\rangle \leqslant O\left(1 / M_{X}\right), \quad\left\langle G_{A B l}\right\rangle \leqslant O\left(1 / M_{X}\right), \\
\left\langle G_{A B C}\right\rangle \leqslant O\left(1 / M_{X}\right), \quad\left\langle G_{A k j}\right\rangle \leqslant O(1 / M), \\
\left\langle G_{A B j}\right\rangle \leqslant O(1 / M), \quad\left\langle G_{A i j}\right\rangle \leqslant O(1 / M)
\end{gathered}
$$

or

$$
\begin{aligned}
& \left\langle W_{\mathrm{SG} A k l}\right\rangle \leqslant O\left(m_{3 / 2} / M_{X}\right), \quad\left\langle W_{\mathrm{SG} A B l}\right\rangle \leqslant O\left(m_{3 / 2} / M_{X}\right), \\
& \left\langle W_{\mathrm{SG} A B C}\right\rangle \leqslant O\left(m_{3 / 2} / M_{X}\right), \quad\left\langle W_{\mathrm{SG} A k j}\right\rangle \leqslant O\left(m_{3 / 2} / M\right), \\
& \left\langle W_{\mathrm{SG} A B j}\right\rangle \leqslant O\left(m_{3 / 2} / M\right), \quad\left\langle W_{\mathrm{SG} A i j}\right\rangle \leqslant O\left(m_{3 / 2} / M\right) .
\end{aligned}
$$

\section{Stability of gauge hierarchy}

The mass-squared matrices of the scalar fields are simply given by the VEV's of the second derivatives of the potential. From Eqs. (29)-(32), we get the relations

$$
\begin{aligned}
V_{I}^{J}= & \frac{\partial^{2} V}{\partial z^{I} \partial z_{J}^{*}} \\
= & M^{2}\left(e^{G / M^{2}}\right)_{I}^{J} U+M^{2}\left(e^{G / M^{2}}\right)_{I} U^{J}+M^{2}\left(e^{G / M^{2}}\right)^{J} U_{I} \\
& +M^{2} e^{G / M^{2}} U_{I}^{J}+\frac{1}{2}\left(\operatorname{Re} f^{-1}\right)_{\alpha \beta, I}^{J} D^{\alpha} D^{\beta} \\
& +\left(\operatorname{Re} f^{-1}\right)_{\alpha \beta, I} D^{\alpha}\left(D^{\beta}\right)^{J}+\left(\operatorname{Re} f^{-1}\right)_{\alpha \beta}^{J} D^{\alpha}\left(D^{\beta}\right)_{I} \\
& +\left(\operatorname{Re} f^{-1}\right)_{\alpha \beta} D^{\alpha}\left(D^{\beta}\right)_{I}^{J}+\left(\operatorname{Re} f^{-1}\right)_{\alpha \beta}\left(D^{\alpha}\right)_{I}\left(D^{\beta}\right)^{J}
\end{aligned}
$$

where the ellipses represent higher order terms in $\Delta z$.

and 


$$
\begin{aligned}
V_{I J}= & \frac{\partial^{2} V}{\partial z^{I} \partial z^{J}} \\
= & M^{2}\left(e^{G / M^{2}}\right)_{I J} U+M^{2}\left(e^{G / M^{2}}\right)_{I} U_{J}+M^{2}\left(e^{G / M^{2}}\right)_{J} U_{I} \\
& +M^{2} e^{G / M^{2}} U_{I J}+\frac{1}{2}\left(\operatorname{Re} f^{-1}\right)_{\alpha \beta, I J} D^{\alpha} D^{\beta} \\
& +\left(\operatorname{Re} f^{-1}\right)_{\alpha \beta, I} D^{\alpha}\left(D^{\beta}\right)_{J}+\left(\operatorname{Re} f^{-1}\right)_{\alpha \beta, J} D^{\alpha}\left(D^{\beta}\right)_{I} \\
& +\left(\operatorname{Re} f^{-1}\right)_{\alpha \beta} D^{\alpha}\left(D^{\beta}\right)_{I J}+\left(\operatorname{Re} f^{-1}\right)_{\alpha \beta}\left(D^{\alpha}\right)_{I}\left(D^{\beta}\right)_{J} .
\end{aligned}
$$

By using the relations (39)-(43), the VEV's of $V_{I}^{J}$ and $V_{I J}$ are estimated as

$$
\begin{gathered}
\left\langle V_{K}^{(F) L}\right\rangle=O\left(M_{X}^{2}\right), \quad\left\langle V_{A}^{(F) B}\right\rangle=O\left(m_{3 / 2}^{2}\right), \\
\left\langle V_{k}^{(F) l}\right\rangle=O\left(m_{3 / 2}^{2}\right), \\
\left\langle V_{i}^{(F) j}\right\rangle=O\left(m_{3 / 2}^{2}\right), \quad\left\langle V_{K}^{(F) B}\right\rangle=O\left(m_{3 / 2} M_{X}\right), \\
\left\langle V_{K}^{(F) l}\right\rangle=O\left(m_{3 / 2} M_{X}\right), \\
\left\langle V_{K}^{(F) j}\right\rangle=O\left(m_{3 / 2} M_{X}^{2} / M\right), \quad\left\langle V_{A}^{(F) l}\right\rangle=O\left(m_{3 / 2}^{2}\right), \\
\left\langle V_{A}^{(F) j}\right\rangle=O\left(m_{3 / 2}^{2} M_{X} / M\right), \quad\left\langle V_{k}^{(F) j}\right\rangle=0
\end{gathered}
$$

and

$$
\begin{gathered}
\left\langle V_{K L}^{(F)}\right\rangle=O\left(m_{3 / 2} M\right), \quad\left\langle V_{A B}^{(F)}\right\rangle=O\left(m_{3 / 2}^{2}\right), \\
\left\langle V_{k l}^{(F)}\right\rangle=O\left(m_{3 / 2} M\right), \\
\left\langle V_{i j}^{(F)}\right\rangle=O\left(m_{3 / 2} M\right), \quad\left\langle V_{K B}^{(F)}\right\rangle=O\left(m_{3 / 2} M\right), \\
\left\langle V_{K l}^{(F)}\right\rangle=O\left(m_{3 / 2} M\right), \\
\left\langle V_{K j}^{(F)}\right\rangle=O\left(m_{3 / 2} M\right), \quad\left\langle V_{A l}^{(F)}\right\rangle=O\left(m_{3 / 2}^{2}\right), \quad\left\langle V_{A j}^{(F)}\right\rangle=O\left(m_{3 / 2}^{2}\right), \\
\left\langle V_{k j}^{(F)}\right\rangle=0,
\end{gathered}
$$

respectively. The quantities of order $m_{3 / 2} M$ in $\left\langle V_{I J}^{(F)}\right\rangle$ originate in the term $\left\langle M e^{G / 2 M^{2}} G_{I J J^{\prime}}\right\rangle\left\langle F^{J^{\prime}}\right\rangle$. If $\left\langle V_{I J}\right\rangle$ 's are $O\left(m_{3 / 2} M\right)$ for the light fields $z^{I}$, the masses of light fields can get intermediate values after the diagonalization of mass matrix. The masses of those fermionic partners stay at the weak scale. The weak scale can be destabilized in the presence of weak Higgs doublets with intermediate masses. This is so called "gauge hierarchy problem." Only when $\left\langle M e^{G / 2 M^{2}} G_{I J J^{\prime}}\right\rangle\left\langle F^{J^{\prime}}\right\rangle$ 's meet some requirements does the hierarchy survive. In this paper, we require the conditions

$$
\left\langle M e^{G / 2 M^{2}} G_{I J J^{\prime}}\right\rangle\left\langle F^{J^{\prime}}\right\rangle \leqslant O\left(m_{3 / 2}^{2}\right)
$$

for the light fields $z^{I}$ and $z^{J}$,

$$
\left\langle M e^{G / 2 M^{2}} G_{I J J^{\prime}}\right\rangle\left\langle F^{J^{\prime}}\right\rangle \leqslant O\left(M_{X}^{2}\right)
$$

for the heavy fields $z^{I}$ and $z^{J}$, and

$$
\begin{gathered}
\left\langle M e^{G / 2 M^{2}} G_{K l J^{\prime}}\right\rangle\left\langle F^{J^{\prime}}\right\rangle \leqslant O\left(m_{3 / 2} M_{X}\right), \\
\left\langle M e^{G / 2 M^{2}} G_{K j J^{\prime}}\right\rangle\left\langle F^{J^{\prime}}\right\rangle \leqslant O\left(m_{3 / 2} M_{X}^{2} / M\right), \\
\left\langle M e^{G / 2 M^{2}} G_{A j J^{\prime}}\right\rangle\left\langle F^{J^{\prime}}\right\rangle \leqslant O\left(m_{3 / 2}^{2} M_{X} / M\right) .
\end{gathered}
$$

The conditions (48) $-(52)$ correspond to the statement that the magnitudes of $\left\langle M e^{G / 2 M^{2}} G_{I J J^{\prime}}\right\rangle\left\langle F^{J^{\prime}}\right\rangle$ are equal to or smaller than the rest terms. The hidden ansatz trivially satisfies the above conditions. The gauge hierarchy problem has been discussed based on the postulation that $M_{X}$ is identified with $M$ in Ref. [28].

The contributions from the $D$ term are naively estimated as

$$
\begin{gathered}
\left\langle V_{\kappa}^{(D) \lambda}\right\rangle=O\left(M_{X}^{2}\right), \quad\left\langle V_{\kappa \lambda}^{(D)}\right\rangle=O\left(M_{X}^{2}\right), \\
\left\langle V_{K}^{(D) j}\right\rangle,\left\langle V_{A}^{(D) j}\right\rangle=O\left(M_{X}^{3} / M\right), \\
\left\langle V_{K j}^{(D)}\right\rangle,\left\langle V_{A j}^{(D)}\right\rangle=O\left(M_{X}^{3} / M\right), \\
\left\langle V_{i}^{(D) j}\right\rangle,\left\langle V_{i j}^{(D)}\right\rangle=O\left(M_{X}^{4} / M^{2}\right), \\
\left\langle V_{k}^{(D) j}\right\rangle,\left\langle V_{k j}^{(D)}\right\rangle=0,
\end{gathered}
$$

where we used the relation $\left\langle\left(D^{A}\right)_{I}\right\rangle=\left\langle\left(z^{\dagger} T^{A}\right)_{\kappa} K_{I}^{\kappa}\right\rangle$ $=O\left(M_{X}\right) K_{I}^{A}$. Under the conditions that $\left\langle V_{I}^{(D) J}\right\rangle \leqslant\left\langle V_{I}^{(F) J}\right\rangle$ and $\left\langle V_{I J}^{(D)}\right\rangle \leqslant\left\langle V_{I J}^{(F)}\right\rangle$ for the light fields $z^{I}$, we obtain relations such as

$$
\left\langle K_{A}^{k}\right\rangle,\left\langle K_{A k}\right\rangle=O\left(\frac{m_{3 / 2}^{2}}{M_{X}^{2}}\right)
$$

and

$$
\left\langle K_{A}^{i}\right\rangle,\left\langle K_{A i}\right\rangle=O\left(\frac{m_{3 / 2}^{2}}{M_{X} M}\right) .
$$

The analysis could be made based on weaker requirements than (48)-(55), but we will not discuss it further to avoid a complication and a subtlety in this paper.

\section{E. Diagonalization of the mass matrix}

The mass term is written as

$$
V^{\text {mass }}=\frac{1}{2}\left\langle V_{\hat{I} \hat{J}}\right\rangle \Delta z^{\hat{I}} \Delta z^{\hat{J}}
$$

where $\quad \Delta z^{\hat{I}}=\left(\Delta z^{K}, \Delta z^{\bar{L}} \equiv \Delta z_{L}^{*} ; \Delta z^{A}, \Delta z^{\bar{B}} \equiv \Delta z_{B}^{*} ; \Delta \tilde{z}^{i}, \Delta \tilde{z}^{\bar{j}} \equiv\right.$ $\left.\Delta \tilde{z}_{j}^{*} ; \Delta z^{k}, \Delta z^{\bar{l}} \equiv \Delta z_{l}^{*}\right)$. From the discussion in the previous subsection, the orders of $\left\langle V_{\hat{I} \hat{J}}\right\rangle$ are estimated as

$$
\left\langle V_{\hat{I} \hat{J}}\right\rangle=O\left(\begin{array}{ccc}
M_{X}^{2} & M_{X}^{2} & m_{3 / 2} M_{X} \\
M_{X}^{2} & M_{X}^{2} & m_{3 / 2}^{2} \\
m_{3 / 2} M_{X} & m_{3 / 2}^{2} & m_{3 / 2}^{2}
\end{array}\right)
$$


for gauge nonsinglet fields $\left(\Delta z^{\hat{K}} ; \Delta z^{\hat{A}} ; \Delta z^{\hat{k}}\right)$ and

$$
\left\langle V_{\hat{I} J}\right\rangle=O\left(\begin{array}{ccc}
M_{X}^{2} & M_{X}^{2} & m_{3 / 2} M_{X}^{2} / M \\
M_{X}^{2} & M_{X}^{2} & m_{3 / 2}^{2} M_{X} / M \\
m_{3 / 2} M_{X}^{2} / M & m_{3 / 2}^{2} M_{X} / M & m_{3 / 2}^{2}
\end{array}\right)
$$

for gauge singlet fields $\left(\Delta z^{\hat{K}} ; \Delta z^{\hat{A}} ; \Delta \tilde{z}^{\hat{i}}\right)$. As the matrix $\left\langle V_{\hat{I} \hat{J}}\right\rangle$ is Hermitian, it can be diagonalized by the use of a certain unitary matrix $U_{\hat{J}}^{\hat{I}}$. The mass eigenstates $\phi^{\hat{I}}$ are related to $\Delta z^{\hat{I}}$ as $\phi^{\hat{I}}=U_{\hat{J}}^{\hat{I}} \Delta z^{\hat{J}}$. We denote the heavy fields with mass $O\left(M_{X}\right)$ as $\phi^{\hat{\mathscr{H}}}$ and the light fields with mass $O\left(m_{3 / 2}\right)$ as $\phi^{\hat{\mathscr{C}}}$ where $\mathscr{H}=(K, A)$ and $\mathscr{L}=(i, k)$. Next we would like to integrate out the heavy fields $\phi^{\hat{\mathscr{H}}}$. For this purpose, it is convenient to choose the variables

$$
\begin{gathered}
\Delta \hat{z}^{\hat{\mathscr{G}}}=\left(U_{\hat{\mathscr{G}} \mathscr{G}^{\prime}}^{\hat{\mathscr{G}}}\right)^{-1} \phi^{\hat{\mathscr{G}}{ }^{\prime}}, \\
\Delta \hat{z}^{\hat{\mathscr{G}}}=\left(U_{\hat{\mathscr{B}}^{\prime}}^{\hat{\mathscr{G}}}\right)^{-1} \phi^{\hat{\mathscr{G}}^{\prime}}
\end{gathered}
$$

or

$$
\begin{gathered}
\Delta \hat{z}^{\hat{I}}=\hat{U}_{\hat{J}}^{\hat{I}} \Delta z^{\hat{J}}, \\
\hat{U}_{\hat{J}}^{\hat{I}} \equiv\left(\begin{array}{cc}
I & \left(U_{\hat{\mathscr{B}}^{\prime}}^{\hat{\mathscr{H}}}\right)^{-1} U_{\hat{\mathscr{C}}}^{\hat{\mathscr{G}}{ }^{\prime}} \\
\left(U_{\hat{\mathscr{C}}^{\prime}}^{\hat{\mathscr{C}}}\right)^{-1} U_{\hat{\mathscr{G}}}^{\hat{\mathscr{C}^{\prime}}} & I
\end{array}\right) .
\end{gathered}
$$

Here we used the fact that $\operatorname{det} U_{\hat{\mathscr{C}} \mathcal{G}^{\prime}}^{\hat{\mathscr{G}}}=1+O\left(m_{3 / 2}^{2} / M_{X}^{2}\right)$ and $\operatorname{det} U_{\hat{\mathscr{S}}^{\prime}}^{\hat{f^{\prime}}}=1+O\left(m_{3 / 2}^{2} / M_{X}^{2}\right)$ and neglected the higher order terms. The orders of off-diagonal elements of $\hat{U}_{\hat{J}}^{\hat{l}}$ are estimated as

$$
\begin{gathered}
\hat{U}_{\hat{l}}^{\hat{K}}=O\left(\frac{m_{3 / 2}}{M_{X}}\right), \quad \hat{U}_{\hat{l}}^{\hat{A}}=O\left(\frac{m_{3 / 2}^{2}}{M_{X}^{2}}\right), \\
\hat{U}_{\hat{j}}^{\hat{K}}=O\left(\frac{m_{3 / 2}}{M}\right), \quad \hat{U}_{\hat{j}}^{\hat{A}}=O\left(\frac{m_{3 / 2}^{2}}{M M_{X}}\right) .
\end{gathered}
$$

\section{F. Calculation of the effective theory}

The rest in the procedure are as follows: (1) We write down the scalar potential by using new variables $\Delta \hat{z}^{\hat{I}}$; (2) We take the flat limit and integrate out the heavy fields by inserting the solutions of the stationary conditions into the full potential.

We can write down the Kähler potential $K$, the superpotential $W_{\mathrm{SG}}$, and the $D$ auxiliary fields $D^{\alpha}$ in terms of the variations $\Delta \hat{z}^{\hat{I}}$ as

$$
K=\langle\hat{K}\rangle+\left\langle\hat{K}_{\hat{I}}\right\rangle \Delta \hat{z}^{\hat{I}}+\frac{1}{2}\left\langle\hat{K}_{\hat{I} \hat{J}}\right\rangle \Delta \hat{z}^{\hat{I}} \Delta \hat{z}^{\hat{J}}+\cdots,
$$

$$
\begin{aligned}
W_{\mathrm{SG}}= & \langle\hat{W}\rangle+\left\langle\hat{W}_{\hat{I}}\right\rangle \Delta \hat{z}^{\hat{I}}+\frac{1}{2}\left\langle\hat{W}_{\hat{I} \hat{J}}\right\rangle \Delta \hat{z}^{\hat{I}} \Delta \hat{z}^{\hat{J}} \\
& +\frac{1}{3 !}\left\langle\hat{W}_{\hat{I} \hat{J} \hat{J}^{\prime}}\right\rangle \Delta \hat{z}^{\hat{I}} \Delta \hat{z}^{\hat{J}} \Delta \hat{z}^{\prime}+\cdots,
\end{aligned}
$$

and

$$
D^{\alpha}=\left(\hat{K}_{\lambda}+\hat{K}_{\hat{I}} \Delta \hat{U}_{\lambda}^{\hat{I}}\right)\left(T^{A}\right)_{\kappa}^{\lambda}\left[\left\langle z^{\kappa}\right\rangle+\left(\hat{U}^{-1}\right)_{\hat{J}}^{\kappa} \Delta \hat{z}^{\hat{J}}\right],
$$

where the ellipses represent terms of higher orders and $\hat{U}_{\hat{J}}^{\hat{I}}=\delta_{\hat{J}}^{\hat{l}}+\Delta \hat{U}_{\hat{J}}^{\hat{I}}$.

For later convenience, we deform $V^{(F)}$ as

$$
\begin{aligned}
V^{(F)}= & \exp \left(\hat{K} / M^{2}\right)\left(\hat{\mathscr{G}}_{\bar{\kappa}}\left(\hat{K}^{-1}\right)^{\bar{\kappa} \lambda} \hat{\mathscr{G}}_{\lambda}\right. \\
& \left.+\hat{\mathscr{G}}_{\bar{i}}\left(\hat{K}^{-1}\right)^{\overline{i j}} \hat{\mathscr{G}}_{j}-3 \frac{|\hat{W}|^{2}}{M^{2}}\right)+\Delta V^{(F)},
\end{aligned}
$$

where

$$
\begin{gathered}
\hat{\mathscr{G}}_{\bar{\kappa}} \equiv \hat{\mathscr{G}}_{\bar{\kappa}}+\hat{\mathscr{S}}_{\bar{i}}\left(\hat{K}^{-1}\right)^{\bar{i} \mu}(\hat{K})_{\mu \bar{\kappa}}, \\
\hat{\mathscr{G}}_{\lambda} \equiv \hat{\mathscr{G}}_{\lambda}+(\hat{K})_{\lambda \bar{\nu}}\left(\hat{K}^{-1}\right)^{\bar{\nu} j} \hat{\mathscr{G}}_{j}, \\
\hat{\mathscr{S}}_{I} \equiv \hat{W}_{\bar{I}}^{*}+\frac{\hat{K}_{\bar{I}}}{M^{2}} \hat{W}^{*}, \\
\hat{\mathscr{G}}_{I} \equiv \hat{W}_{I}+\frac{\hat{K}_{I}}{M^{2}} \hat{W}
\end{gathered}
$$

and

$$
\begin{aligned}
\left(\hat{K}^{-1}\right)^{\bar{i} j} \equiv & \left(\hat{K}^{-1}\right)^{\bar{i} j}-\left(\hat{K}^{-1}\right)^{\bar{i} \mu}(\hat{K})_{\mu \nu}\left(\hat{K}^{-1}\right)^{\bar{\nu} j}, \\
\Delta V^{(F) \equiv} \equiv & \exp \left(\hat{K} / M^{2}\right)\left\{\hat{\mathscr{G}}_{\hat{I}} \Delta(\hat{U})_{\bar{I}}^{\hat{I}}\left(\hat{K}^{-1}\right)^{\bar{I} J} \hat{\mathscr{G}}_{J}\right. \\
& +\hat{\mathscr{G}}_{\bar{I}}\left(\hat{K}^{-1}\right)^{\bar{I} J} \hat{\mathscr{G}}_{\hat{J}} \Delta(\hat{U})_{J}^{\hat{J}} \\
& +\hat{\mathscr{G}}_{\bar{I}}\left[\left(\hat{K}^{-1}\right)^{\hat{J} J} \Delta\left(\hat{U}^{-1}\right)_{\hat{J}}^{\bar{I}}\right. \\
& \left.\left.+\left(\hat{K}^{-1}\right)^{\hat{I} \bar{I}} \Delta\left(\hat{U}^{-1}\right)_{\hat{I}}^{J}\right] \hat{\mathscr{G}}_{J}\right\} \\
& +O\left(\left(\Delta U^{(-1)}\right)^{2}\right) .
\end{aligned}
$$

We should not confuse $\left(\hat{K}^{-1}\right)^{\overline{i j}}, \hat{\mathscr{G}}_{\bar{\kappa}}$, and $\hat{\mathscr{G}}_{\lambda}$ with $\left(\hat{K}^{-1}\right)^{\bar{i} j}, \hat{\mathscr{G}}_{\bar{K}}$, and $\hat{\mathscr{G}}_{\lambda}$, respectively. (Note the difference of the size of the caret.) Here $(\hat{K})_{\mu \bar{\nu}}$ is the inverse matrix of $\left(\hat{K}^{-1}\right)^{\mu \bar{\nu}}$. The scalar mass matrix is diagonalized up to 
$O\left(m_{3 / 2} / M_{X}\right)$ in terms of $\Delta \hat{z}^{\hat{I}}$, and so the fields $\Delta \hat{z}^{\hat{\kappa}}$ can be regarded as properly normalized fields and

$$
\left\langle\left(\hat{K}^{-1}\right)^{\bar{\kappa} \lambda}\right\rangle=\delta^{\bar{\kappa} \lambda}\left[1+O\left(\frac{m_{3 / 2}^{2}}{M_{X}^{2}}\right)\right]
$$

up to off-diagonal element of order $O\left(m_{3 / 2} / M_{X}\right)$ after the scale transformation is made. We expand $\Delta \hat{z}^{\hat{I}}$ in powers of $m_{3 / 2}$ such as

$$
\Delta \hat{z}^{\hat{I}}=\delta \hat{z}^{\hat{I}}+\delta^{2} \hat{z}^{\hat{I}}+\cdots,
$$

with $\delta^{n} \hat{z}^{\hat{I}}=O\left(m_{3 / 2}^{n} / M_{X}^{n-1}\right)$ and $\Delta \hat{z}^{\hat{k}}=O\left(m_{3 / 2}\right)$ for the light fields, e.g., $\delta^{2} \hat{z}^{\hat{k}}=\delta^{3} \hat{z}^{\hat{k}}=\cdots=0$. In the same way, we expand the $\hat{\mathscr{G}}_{\hat{\lambda}}, \hat{\mathscr{G}}_{\hat{j}}$, and $\hat{D}^{\alpha}$ in powers of $m_{3 / 2}$ such as

$$
\begin{aligned}
& \hat{\mathscr{G}}_{\hat{\lambda}}=\delta \hat{\mathscr{G}}_{\hat{\lambda}}+\delta^{2} \hat{\mathscr{G}}_{\hat{\lambda}}+\cdots, \\
& \hat{\mathscr{G}}_{\hat{j}}=\delta \hat{\mathscr{G}}_{\hat{j}}+\delta^{2} \hat{\mathscr{G}}_{\hat{j}}+\cdots,
\end{aligned}
$$

and

$$
\hat{D}^{\alpha}=\delta \hat{D}^{\alpha}+\delta^{2} \hat{D}^{\alpha}+\cdots
$$

Those orders are given as $\delta^{n} \hat{\mathscr{G}}_{\hat{\lambda}}=O\left(m_{3 / 2}^{n} / M_{X}^{n-2}\right)$, $\delta^{n} \hat{G}_{j}=O\left(m_{3 / 2}^{n} / M^{n-2}\right)$, and $\delta^{n} \hat{D}^{\alpha}=O\left(m_{3 / 2}^{n} / M_{X}^{n-2}\right)$ up to the factor $O\left(\left(M_{X} / M\right)^{n}\right)$.

The following relations are derived from the expansions of $\hat{\mathscr{G}}_{\lambda}$ and $\hat{\mathscr{G}}_{j}$ :

$$
\begin{gathered}
\delta \hat{\mathscr{G}}_{K}=\left\langle\hat{W}_{K}\right\rangle+\left\langle\hat{W}_{K L}\right\rangle \delta \hat{z}^{L}+\frac{\langle\hat{W}\rangle}{M^{2}}\left\langle\hat{K}_{K}\right\rangle \\
+\left\langle(\hat{K})_{K \bar{\nu}}\right\rangle\left\langle\left(\hat{K}^{-1}\right)^{\bar{\nu} j}\right\rangle \delta \hat{\mathscr{G}}_{j}, \\
\delta^{2} \hat{\mathscr{G}}_{K}=\left\langle\hat{W}_{K L}\right\rangle \delta^{2} \hat{z}^{L}+\left\langle\hat{W}_{K \mathscr{L}}\right\rangle \delta \hat{z}^{\mathscr{\mathscr { C }}}+\left\langle\hat{W}_{K A}\right\rangle \delta \hat{z}^{A} \\
+\frac{1}{2}\left\langle\hat{W}_{K \lambda \mu}\right\rangle \delta \hat{z}^{\lambda} \delta \hat{z}^{\mu} \\
+\frac{1}{M^{2}}\left(\frac{1}{2}\left\langle\hat{W}_{L M}\right\rangle \delta \hat{z}^{L} \delta \hat{z}^{M}\left\langle\hat{K}_{K}\right\rangle+\langle\hat{W}\rangle\left\langle\hat{K}_{K \hat{J}}\right\rangle \delta \hat{z}^{\hat{J}}\right) \\
+\left\langle(\hat{K})_{K \bar{\nu}}\right\rangle\left\langle\left(\hat{K}^{-1}\right)^{\bar{\nu} j}\right\rangle \delta^{2} \hat{\mathscr{G}}_{j}+\delta\left((\hat{K})_{K \bar{\nu}}\left(\hat{K}^{-1}\right)^{\bar{\nu} j}\right) \delta \hat{\mathscr{G}}_{j},
\end{gathered}
$$

$$
\begin{aligned}
\delta^{2} \hat{\mathscr{G}}_{A}= & \left\langle\hat{W}_{A I}\right\rangle \delta \hat{z}^{I}+\frac{1}{2}\left\langle\hat{W}_{A K I}\right\rangle \delta \hat{z}^{K} \delta \hat{z}^{I}+\frac{\langle\hat{W}\rangle}{M^{2}}\left\langle\hat{K}_{A \hat{J}}\right\rangle \delta \hat{z}^{\hat{J}} \delta \\
& +\left\langle(\hat{K})_{A \bar{\nu}}\right\rangle\left\langle\left(\hat{K}^{-1}\right)^{\bar{\nu} j}\right\rangle \delta^{2} \hat{\mathscr{G}}_{j}+\delta\left((\hat{K})_{A \bar{\nu}}\left(\hat{K}^{-1}\right)^{\bar{\nu} j}\right) \delta \hat{\mathscr{G}}_{j}
\end{aligned}
$$

and

$$
\begin{gathered}
\delta \hat{\mathscr{G}}_{j}=\left\langle\hat{W}_{j}\right\rangle+\frac{\langle\hat{W}\rangle}{M^{2}}\left\langle\hat{K}_{j}\right\rangle, \\
\delta^{2} \hat{\mathscr{G}}_{j}=\left\langle\hat{W}_{j I}\right\rangle \delta \hat{z}^{I}+\frac{1}{2}\left\langle\hat{W}_{j I J}\right\rangle \delta \hat{z}^{I} \delta \hat{z}^{J} \\
+\frac{1}{M^{2}}\left(\frac{1}{2}\left\langle\hat{W}_{L M}\right\rangle \delta \hat{z}^{L} \delta \hat{z}^{M}\left\langle\hat{K}_{j}\right\rangle+\langle\hat{W}\rangle\left\langle\hat{K}_{j \hat{J}}\right\rangle \delta \hat{z}^{\hat{J}}\right),
\end{gathered}
$$

respectively. While the expansion of $\hat{D}^{A}$ gives

$$
\begin{aligned}
\delta \hat{D}^{A}= & \left\langle\hat{K}_{\lambda}\right\rangle\left(T^{A}\right)_{\kappa}^{\lambda} \delta \hat{z}^{\kappa} \\
& +\left(\left\langle\hat{K}_{\hat{I} \lambda}\right\rangle \delta \hat{z}^{\hat{I}}+\left\langle\hat{K}_{\hat{I}}\right\rangle \delta \hat{U}_{\lambda}^{\hat{I}}\right)\left(T^{A}\right)_{\kappa}^{\lambda}\left\langle z^{\kappa}\right\rangle,
\end{aligned}
$$

$$
\delta^{2} \hat{D}^{A}=\left\langle\hat{K}_{\lambda}\right\rangle\left(T^{A}\right)_{\kappa}^{\lambda}\left[\delta^{2} \hat{z}^{\kappa}+\delta\left(\hat{U}^{-1}\right)_{\hat{J}}^{\kappa} \delta \hat{z}^{\hat{J}}\right]
$$

$$
\begin{aligned}
& +\left(\left\langle\hat{K}_{\hat{I} \lambda}\right\rangle \delta^{2} \hat{z}^{\hat{I}}+\left\langle\hat{K}_{\hat{I} \hat{J} \lambda}\right\rangle \delta \hat{z}^{\hat{I}} \delta \hat{z}^{\hat{J}}\right. \\
& \left.+\left\langle\hat{K}_{\hat{I}}\right\rangle \delta^{2} \hat{U}_{\lambda}^{\hat{I}}\right)\left(T^{A}\right)_{\kappa}^{\lambda}\left\langle z^{\kappa}\right\rangle \\
& +\left(\left\langle\hat{K}_{\hat{I} \lambda}\right\rangle \delta \hat{z}^{\hat{I}}+\left\langle\hat{K}_{\hat{I}}\right\rangle \delta \hat{U}_{\lambda}^{\hat{I}}\right)\left(T^{A}\right)_{\kappa}^{\lambda} \delta \hat{z}^{\kappa} .
\end{aligned}
$$

The stationary conditions $\partial V / \partial z^{K}=0$ and $\partial V / \partial z^{A}=0$ give

$$
\langle\hat{W}\rangle_{K L}\left\langle\left(\hat{K}^{-1}\right)^{L \bar{\mu}}\right\rangle \delta \hat{\mathscr{S}}_{\bar{\mu}}=0,
$$

$$
\begin{aligned}
\langle\hat{W}\rangle_{K L}\left\langle\left(\hat{K}^{-1}\right)^{L \bar{\mu}}\right\rangle \delta^{2} \hat{\mathscr{G}}_{\bar{\mu}}= & -\delta \hat{\mathscr{G}}_{\bar{\mu}}\left\langle\left(\hat{K}^{-1}\right)^{\bar{\mu} \lambda}\right\rangle\left\langle\hat{W}_{\lambda \sigma K}\right\rangle \delta \hat{z}^{\sigma} \\
& + \text { const }
\end{aligned}
$$

and

$$
\delta \hat{\mathscr{G}}_{A}=\left\langle(\hat{K})_{A \bar{\nu}}\right\rangle\left\langle\left(\hat{K}^{-1}\right)^{\bar{\nu} j}\right\rangle \delta \hat{\mathscr{G}}_{j},
$$

$$
\left\langle\operatorname{Re} f_{\alpha \beta}^{-1}\right\rangle\left\langle\left(\hat{z} T^{\alpha}\right)^{\bar{\mu}}\right\rangle\left\langle\hat{K}_{A \bar{\mu}}\right\rangle \delta \hat{D}^{\beta}=0,
$$




$$
\begin{aligned}
\left\langle\operatorname{Re} f_{\alpha \beta}^{-1}\right\rangle\left\langle\left(\hat{z} T^{\alpha}\right)^{\bar{\mu}}\right\rangle\left\langle\hat{K}_{A \bar{\mu}}\right\rangle \delta^{2} D^{\beta}= & E \delta \hat{\mathscr{C}}_{\bar{\mu}}\left\langle\left(K^{-1}\right)^{\bar{\mu} \lambda}\right\rangle \\
& \times\left\langle\hat{W}_{\lambda \sigma A}\right\rangle \delta \hat{z}^{\sigma}+\mathrm{const}
\end{aligned}
$$

respectively. Here $E \equiv\left\langle\exp \left(K / M^{2}\right)\right\rangle$.

From Eqs. (80), (82), (84), and (88), we find $\delta \hat{z}^{K}=0$ by using $\left\langle\delta \hat{z}^{K}\right\rangle=0$. Equation (89) gives the solution for $\delta^{2} \hat{\mathscr{G}}_{\bar{K}}$ as

$$
\begin{aligned}
\delta^{2} \hat{\mathscr{G}}_{\bar{K}}= & \left\langle\hat{\mathscr{G}}_{\bar{K}}\right\rangle-\left\langle\left(\hat{K}_{\bar{K} L}\right)\right\rangle\left\langle\hat{W}^{-1}\right\rangle{ }^{K L} \delta \hat{\mathscr{G}}_{\bar{\mu}}\left\langle\left(K^{-1}\right)^{\bar{\mu} M}\right\rangle \\
& \times\left\langle\hat{W}_{M K l}\right\rangle \delta \hat{z}^{l},
\end{aligned}
$$

where a constant factor of $\delta^{2} \hat{\mathscr{G}}_{\bar{K}}$ is denoted as $\left\langle\hat{\mathscr{G}}_{\bar{K}}\right\rangle$. From Eqs. (64), (86), and (90), we find $\delta \hat{D}^{A}=0$ and $\delta \hat{z}^{A}=0$. By using the relations $\left\langle\hat{W}_{A B k}\right\rangle=O\left(m_{3 / 2} / M_{X}\right)$ and $\left\langle\hat{W}_{A B i}\right\rangle$ $=O\left(m_{3 / 2} / M\right)$, we can show that $\delta^{2} \hat{D}^{A}$ is a constant independent of the light fields. Therefore we will denote it by $\left\langle\hat{D}^{A}\right\rangle$.

Now it is straightforward to calculate the scalar potential $\mathscr{V}^{\text {eff }}$ in the low-energy effective theory by substituting the solutions of the stationary conditions for the heavy fields. The result can be compactly expressed if we define the effective superpotential $\hat{\mathscr{W}}_{\text {eff }}$ as

$$
\hat{\mathscr{W}}_{\mathrm{eff}}(z)=\frac{1}{2 !} \hat{\mu}_{k l} \delta \hat{z}^{k} \delta \hat{z}^{l}+\frac{1}{3 !} \hat{h}_{k l m} \delta \hat{z}^{k} \delta \hat{z}^{l} \delta \hat{z}^{m}
$$

where

$$
\begin{aligned}
\hat{\mu}_{k l} \equiv & E^{1 / 2}\left(\left\langle\hat{W}_{k l}\right\rangle+\frac{\langle\hat{W}\rangle}{M^{2}}\left\langle\hat{K}_{k l}\right\rangle-\left\langle\hat{K}_{k l i}\right\rangle\left\langle\left(\hat{K}^{-1}\right)^{\bar{i} j}\right\rangle \delta \hat{\mathscr{G}}_{j}\right) \\
& +\left(m^{\prime \prime \prime}{ }_{3 / 2}\right)_{k l}, \\
& \hat{h}_{k l m} \equiv E^{1 / 2}\left\langle\hat{W}_{k l m}\right\rangle .
\end{aligned}
$$

Then we can write down the scalar potential of effective theory as ${ }^{10}$

$$
\begin{gathered}
\mathscr{T}_{\mathrm{eff}}^{\mathrm{eff}}=\mathscr{T}_{\mathrm{SUSY}}^{\mathrm{eff}}+\mathscr{T}_{\mathrm{soft}}^{\mathrm{eff}}, \\
\mathscr{T}_{\mathrm{SUSY}}^{\mathrm{eff}}=\left|\frac{\partial \hat{\mathscr{V}}_{\mathrm{eff}}}{\partial \hat{z}^{k}}\right|^{2}+\frac{1}{2} g_{a}^{2}\left[\hat{z}^{\bar{k}}\left(T^{a}\right)_{\bar{k} l} \hat{z}^{l}\right]^{2},
\end{gathered}
$$

\footnotetext{
${ }^{10}$ Here we omitted the terms irrelevant to the gauge nonsinglet fields $\delta \hat{z}^{\hat{k}}$ and the terms whose magnitudes are less than $O\left(m_{3 / 2}^{4}\right)$.
}

$$
\begin{aligned}
\mathscr{\mathscr { T }}_{\mathrm{soft}}^{\mathrm{eff}}= & A \hat{\mathscr{W}}_{\mathrm{eff}}+B^{k}(\hat{z})_{\mathrm{eff}} \frac{\partial \hat{\mathscr{W}}_{\mathrm{eff}}}{\partial \hat{z}^{k}}+\text { H.c. } \\
& +B^{k}(\hat{z})_{\mathrm{eff}} B_{k}(\hat{z})_{\mathrm{eff}}+C(\hat{z})_{\mathrm{eff}} \\
& +\Delta \hat{\mathscr{V}}+\Delta \mathscr{\mathscr { V }}^{\prime(F)},
\end{aligned}
$$

where $\Delta \hat{\mathscr{V}}+\Delta \mathscr{\mathscr { V }}^{\prime(F)}$ is a sum of contributions such as

$$
\Delta \hat{\mathscr{V}}=\Delta \hat{\mathscr{V}}_{0}^{(F)}+\Delta \hat{\mathscr{V}}_{0}^{(D)}+\Delta \hat{\mathscr{V}}_{1}^{(F)}+\Delta \hat{\mathscr{V}}_{1}^{(D)}
$$

$$
\begin{aligned}
\Delta \hat{\mathscr{V}}_{0}^{(F)} \equiv & E\left\{-\left|\delta^{2} \hat{\mathscr{G}}_{K}\right|^{2}+\left|\delta \delta^{2} \hat{\mathscr{G}}_{A}\right|^{2}+\delta \hat{\mathscr{G}}_{\bar{A}} \delta^{\bar{A} B} \delta^{3^{\prime} \hat{\mathscr{G}}_{B}+\text { H.c. }}\right. \\
& +\delta^{2} \hat{\mathscr{G}}_{\bar{\kappa}} \delta^{\bar{\kappa} L}\left(\left\langle\hat{W}_{L k}\right\rangle \delta \hat{z}^{k}+\frac{1}{2}\left\langle\hat{W}_{L k l}\right\rangle \delta \hat{z}^{k} \delta \hat{z}^{l}+\cdots\right) \\
& + \text { H.c. }\}
\end{aligned}
$$

$$
\Delta \hat{\mathscr{V}}_{0}^{(D)} \equiv\left\langle\operatorname{Re} f_{A B}^{-1}\right\rangle\left\langle\hat{D}^{A}\right\rangle\left\langle\hat{K}_{\hat{I} \lambda}\right\rangle \delta \hat{z}^{\hat{I}}\left(T^{B}\right)_{\kappa}^{\lambda} \delta \hat{z}^{\kappa}
$$

$$
\Delta \hat{\mathscr{V}}_{1}^{(F)} \equiv E\left\{\delta \hat{\mathscr{G}}_{\bar{\kappa}} \delta^{2^{\prime}}\left(\hat{K}^{-1}\right)^{\bar{\kappa} \lambda} \delta \hat{\mathscr{G}}_{\lambda}+\delta \hat{\mathscr{G}}_{\bar{\kappa}} \delta\left(\hat{K}^{-1}\right)^{\bar{\kappa} K} \delta^{2^{\prime}} \hat{\mathscr{G}}_{K}\right.
$$$$
+ \text { H.c.\}, }
$$

$$
\Delta \hat{\mathscr{V}}_{1}^{(D)} \equiv\left\langle\operatorname{Re} f_{A B}^{-1}\right\rangle\left\langle\hat{D}^{A}\right\rangle\left\langle\hat{K}_{\hat{I} \hat{J} \lambda}\right\rangle \delta \hat{z}^{\hat{I}} \delta \hat{z}^{\hat{J}}\left(T^{B}\right)_{\kappa}^{\lambda}\left\langle z^{\kappa}\right\rangle
$$

$$
\begin{aligned}
E[\text { const }]^{L} \equiv & E\left\{\delta \hat{\mathscr{C}}_{\hat{I}} \delta(\hat{U})_{\bar{I}}^{\hat{I}_{I}}\left\langle\left(\hat{K}^{-1}\right)^{\bar{I} L}\right\rangle+\delta \hat{\mathscr{G}}_{\bar{I}}\left\langle\left(\hat{K}^{-1}\right)^{\bar{I} J}\right\rangle \delta(\hat{U})_{J}^{L}\right. \\
& +\delta \hat{\mathscr{C}}_{\bar{I}}\left[\left\langle\left(\hat{K}^{-1}\right)^{\hat{J} L}\right\rangle \delta\left(\hat{U}^{-1}\right)_{\hat{J}}^{\bar{I}}\right. \\
& \left.\left.+\left\langle\left(\hat{K}^{-1}\right)^{\hat{I} \bar{I}}\right\rangle \delta\left(\hat{U}^{-1}\right)_{\hat{I}}^{L}\right]\right\} .
\end{aligned}
$$

The quantities with a prime such as $\delta^{3^{\prime}} \hat{\mathscr{G}}_{B}$ mean that the terms proportional to $\delta^{2} \hat{z}^{\hat{I}}$ are omitted. The ellipsis in Eq. (100) represents other terms in $\delta^{2} \hat{\mathscr{G}}_{K}-\left\langle\hat{W}_{K L}\right\rangle \delta^{2} \hat{z}^{L}$. [Refer to Eq. (81).] The parameters $A, B^{\bar{k}}(z)_{\mathrm{eff}}$, and $C(\hat{z})_{\mathrm{eff}}$ are given as 


$$
\begin{gathered}
A=m_{3 / 2}^{*}-3 m_{3 / 2}^{*}, \\
B^{k}(\hat{z})_{\mathrm{eff}}=\left(m_{3 / 2}^{*}+m_{3 / 2}^{* \prime \prime}+m_{3 / 2}^{* \prime \prime \prime}\right)_{k l}^{\bar{k} l} \delta^{\bar{k} k} \delta \hat{z}^{l}, \\
C(\hat{z})_{\mathrm{eff}}=E \delta \hat{\mathscr{G}}_{\bar{i}}\left\langle\left(\hat{K}^{-1}\right)^{\bar{i} j}\right\rangle\left(\frac{1}{3 !}\left\langle\hat{W}_{j I J J^{\prime}}\right\rangle \delta \hat{z}^{I} \delta \hat{z}^{J} \delta \hat{z}^{J^{\prime}}+\frac{\left\langle\hat{W}^{\prime}\right.}{M^{2}} \delta^{2^{\prime}} \hat{K}_{j}\right)+\text { H.c. }+E\left(\delta \hat{\mathscr{G}}_{\bar{i}} \delta^{2^{\prime}}\left(\hat{K}^{-1}\right)^{\bar{i} j} \delta \hat{\mathscr{G}}_{j}+\frac{\langle V\rangle}{M^{2}} \delta^{2^{\prime}} \hat{K}\right) \\
-\left\{\left(m_{3 / 2}^{* \prime \prime \prime}\right)_{l i}\left(m_{3 / 2}^{\prime \prime \prime}\right)_{k \bar{k}}+\left(m_{3 / 2}^{\prime \prime \prime}\right)_{k l}\left(m_{3 / 2}^{* \prime \prime}\right)_{\bar{k} l} \bar{l}\right\} \delta^{k \bar{l}} \delta \hat{z}^{\bar{k}} \delta \hat{z}^{l}-\left\{\left(m_{3 / 2}^{\prime \prime \prime}\right)_{m l} \delta^{m \bar{k}}\left(m_{3 / 2}^{*}+m_{3 / 2}^{*}\right)_{\bar{k} k} \delta \hat{z}^{k} \delta \hat{z}^{l}+\mathrm{H} . \mathrm{c} .\right\} \\
+A\left[E^{1 / 2}\left(\frac{\left\langle\hat{W}^{2}\right.}{M^{2}}\left\langle\hat{K}_{k l}\right\rangle-\left\langle\hat{K}_{k l i}\right\rangle\left\langle\left(\hat{K}^{-1}\right)^{\bar{i} j}\right\rangle \delta \hat{\mathscr{G}}_{j}\right)+\left(m_{3 / 2}^{\prime \prime \prime}\right)_{k l}\right] \delta \hat{z}^{k} \delta \hat{z}^{l},
\end{gathered}
$$

where

$$
\begin{gathered}
\left(m_{3 / 2}\right)_{k \bar{l}}=E^{1 / 2} \frac{\langle\hat{W}\rangle}{M^{2}} \delta_{k \bar{l}}, \\
m_{3 / 2}^{\prime}=E^{1 / 2} \frac{\left\langle\hat{K}_{\bar{i}}\right\rangle}{M^{2}}\left\langle\left(\hat{K}^{-1}\right)^{\bar{i} j}\right\rangle \delta \hat{\mathscr{G}}_{j}, \\
\left(m_{3 / 2}^{\prime \prime}\right)_{k \bar{l}}=-E^{1 / 2}\left\langle\hat{K}_{k \bar{l} \bar{i}}\right\rangle\left\langle\left(\hat{K}^{-1}\right)^{\bar{i} j}\right\rangle \delta \hat{\mathscr{G}}_{j}, \\
\left(m_{3 / 2}^{\prime \prime \prime}\right)_{\kappa \hat{l}}=-E^{1 / 2}\left\langle\hat{K}_{\kappa} \hat{\bar{l}} \overline{\bar{A}}\right\rangle\left\langle\left(\hat{K}^{-1}\right)^{\bar{A} \lambda}\right\rangle \delta \hat{\mathscr{G}}_{\lambda} .
\end{gathered}
$$

The $\Delta \hat{\mathscr{V}}_{0}^{(D)}$ and $\Delta \hat{\mathscr{V}}_{1}^{(D)}$ come from the $D$ term of the heavy gauge sector and are referred to as the $D$-term contributions, while the others are called the $F$-term contributions.

The scalar potential obtained should be regarded as the effective theory renormalized at the scale $M_{X}$. This potential serves a matching condition when we solve one-loop renormalization group equations above and below the scale $M_{X}$. The potential is written in terms of SUGRA, and so it will be useful to disclose the structure of SUGRA from the measurement of the SUSY spectrum.

We should consider the renormalization effects for the soft SUSY-breaking parameters and diagonalize the scalar mass matrix $\left\langle V_{\hat{k}}\right\rangle$ after $\mathrm{SU}(2)_{L} \times \mathrm{U}(1)_{Y}$ breaking to derive the weak scale SUSY spectrum.

\section{FEATURES OF THE EFFECTIVE LAGRANGIAN}

The effective theory obtained in the previous section has some excellent features. We discuss two topics.

\section{A. Chirality-conserving mass}

Let us discuss a chirality-conserving mass $\left(m^{2}\right)_{k \bar{l}}$, namely, the coefficient of $\delta \hat{z}^{k} \delta \hat{z}^{\bar{l}}$. They are easily extracted from $\mathscr{T}$ soft and given by

$$
\begin{gathered}
\left(m^{2}\right)_{k \bar{l}}=\left(m_{0}^{2}\right)_{k \bar{l}}+\left(\Delta \hat{\mathscr{V}}_{0}\right)_{k \bar{l}}+\left(\Delta \hat{\mathscr{V}}_{1}\right)_{k \bar{l}} \\
\left(m_{0}^{2}\right)_{k \bar{l}} \equiv \frac{\partial}{\partial \hat{z}^{k}} B^{m}(\hat{z})_{\mathrm{eff}} \frac{\partial}{\partial \hat{z}^{l}} B_{m}(\hat{z})_{\mathrm{eff}}+\frac{\partial^{2}}{\partial \hat{z}^{k} \partial \hat{z}^{l}} C(\hat{z})_{\mathrm{eff}} \\
\left(\Delta \hat{\mathscr{V}}_{0}\right)_{k \bar{l}} \equiv \frac{\partial^{2}}{\partial \hat{z}^{k} \partial \hat{z}^{l}} \Delta \hat{\mathscr{V}}_{0}^{(F)}+\left\langle\operatorname{Re} f_{A B}^{-1}\right\rangle\left\langle\hat{D}^{A}\right\rangle\left(T^{B}\right)_{k \bar{l}}
\end{gathered}
$$

$\left(\Delta \hat{\mathscr{V}}_{1}\right)_{k \bar{l}} \equiv \frac{\partial^{2}}{\partial \hat{z}^{k} \partial \hat{z}^{l}} \Delta \hat{\mathscr{V}}_{1}^{(F)}+2\left\langle\operatorname{Re} f_{A B}^{-1}\right\rangle\left\langle\hat{D}^{A}\right\rangle\left\langle\hat{K}_{k \bar{l} \lambda}\right\rangle\left(T^{B}\right)_{\kappa}^{\lambda}\left\langle z^{\kappa}\right\rangle$.

The term $\left(m_{0}^{2}\right)_{k \bar{l}}$ is present before the heavy sector is integrated out and so it respects the original unified gauge symmetry. On the other hand, other terms coming from $\Delta \hat{\mathscr{V}}$ can pick out effects of the symmetry breaking.

The last terms in Eqs. (115) and (116) are the $D$-term contributions. We discuss the conditions of their existence. The nonzero VEV of the $D$ term is allowed for a $\mathrm{U}(1)$ factor, i.e., a diagonal generator from the gauge invariance. And the $D$ term for an unbroken generator cannot have its VEV. Thus it can arise when the rank of the gauge group is reduced by the gauge symmetry breaking. The $D$-term contribution is proportional to the charge of the broken $\mathrm{U}(1)$ factor and gives mass splittings within the same multiplet in the full theory. We can rewrite $\delta^{2} \hat{D}^{A}=\left\langle\hat{D}^{A}\right\rangle$ as

$$
\left\langle\hat{D}^{A}\right\rangle=2\left(M_{V}^{-2}\right)^{A B} E \delta \hat{\mathscr{G}}_{\kappa} \delta \hat{\mathscr{C}}_{\hat{\lambda}}^{-}\left\{G_{\bar{\mu}}^{\kappa \bar{\lambda}}\left(\hat{z} T^{B}\right)^{\bar{\mu}}+G^{\bar{\mu} \kappa}\left(T^{B}\right)_{\bar{\mu}}^{\bar{\lambda}}\right\}
$$

by using the gauge invariance. We can see that the VEV's vanish up to $O\left(m_{3 / 2}^{4} / M_{X}^{2}\right)$ at $M$ when the Kähler potential 
has a minimal structure. ${ }^{11}$ Hence a sizable $D$-term contribution can appear at $M$ when the Kähler potential has a nonminimal structure.

The other terms in Eqs. (115) and (116) are related to the $F$ terms. They can be neglected in the case that the superpotential couplings are weak and $R$-parity conservation is assumed. Therefore phenomenologically the $D$-term contribution to the scalar masses is important to probe SUSY-GUT models because it can give an additional contribution to squarks, sleptons, and Higgs bosons [17].

\section{B. Specific case}

Finally we discuss the relation between our result and that in Ref. [14]. For later convenience, we list features in the approach of Ref. [14].

(1) The starting theory is a unified theory obtained by taking the flat limit of SUGRA with a certain type of total Kähler potential, and so terms of order $m_{3 / 2}^{4}\left(M_{X} / M\right)^{n}$ are neglected. Since the unification scale $M_{X}$ is now believed to be lower than the gravitational scale $M$ from LEP data [11], this procedure can be justified in such a model. However, it will be important when higher order corrections are to be considered. Then we must incorporate threshold effects and loop effects.

(2) The hidden assumption on the superpotential was taken because it was proposed to discuss consequences independent of the details of each model. The stability of the gauge hierarchy is automatically guaranteed under this assumption.

(3) Heavy-light mixing, in general, can occur after soft SUSY-breaking terms are incorporated. Then we must redefine the scalar fields by diagonalizing the mass matrix. It was assumed that there is no heavy-light mixing after the SUSY breaking.

We shall derive the previous one, $V^{\text {eff(non) }}$, from our scalar potential $\mathscr{T}^{\text {eff }}$ by referring to the list.

(1) When we take the limit $M_{X} / M \rightarrow 0$, we find that some terms vanish. For example, $\left(m_{3 / 2}^{\prime \prime \prime}\right)_{\kappa} \hat{l}$ and $\Delta \hat{\mathscr{V}}_{1}$ vanish.

(2) We take the hidden ansatz $\left\langle\hat{W}_{j} \ldots k \ldots\right\rangle=0$. Then the trilinear coupling constant is reduced to

$$
A E^{1 / 2}\left\langle\hat{W}_{k l m}\right\rangle+\frac{1}{2}\left(m_{3 / 2}^{*}+m_{3 / 2}^{* \prime \prime}\right)_{k \bar{k}} \delta^{\bar{k} n}\left\langle\hat{W}_{n l m}\right\rangle
$$

(3) When we take a model with no heavy-light mixing, $\Delta \mathscr{V}^{\prime(F)}$ does not exist. We can find an ansatz for the Kähler potential that the heavy-light mixing does not occur in the gauge nonsinglet sector after taking the flat limit. For example, the ansatz

$$
K=K^{(\mathscr{H})}\left(z^{\mathscr{H}}, z_{\mathscr{H}}^{*} ; \tilde{z}, \tilde{z}^{*}\right)+K^{(k)}\left(z^{k}, z_{k}^{*} ; \tilde{z}, \tilde{z}^{*}\right)
$$

fulfills our requirement.

We find that $\mathscr{V}^{\text {eff }}$ reduces to $V^{\text {eff(non) }}$, after the above procedures.

\footnotetext{
${ }^{11}$ The $D$-term contributions can be sizable at $M_{X}$ by the radiative correction even when they vanish at $M$.
}

\section{CONCLUSIONS}

We have derived the low-energy effective Lagrangian from SUGRA with nonminimal structure and unified gauge symmetry under some physical assumptions and requirements in a model-independent manner. We have calculated the scalar potential by taking the flat limit and integrating out the heavy sector. The result is summarized in Eqs. (93)(112). The potential is written down in terms of SUGRA, and so it will be useful to disclose the structure of SUGRA from measurement of the SUSY spectrum. We state our chief results in correspondence with the assumptions.

We have assumed that the starting SUGRA consists of a nonminimal Kälher potential and a superpotential without the hidden ansatz. It is important to investigate its consequences at low energy because nonminimal SUGRA appears naturally in many circumstanses. For example, SST's lead to nonminimal SUGRA's effectively. Even if SUGRA has the minimal structure at the tree level, it can get renormalized and as a result, in general, become nonminimal. The nonminimality leads to nonuniversal soft SUSY-breaking terms as pointed out in Ref. [21]. The dangerous $B$ term, which destabilizes the weak scale, can exist if any conditions are not imposed on Yukawa couplings in the superpotential.

We have assumed that SUGRA has unified gauge symmetry which is broken down at $M_{X}$. Some scalar fields get the VEV's of $O\left(M_{X}\right)$. There exist heavy fields with masses of $O\left(M_{X}\right)$ in addition to light fields with masses of $O\left(m_{3 / 2}\right)$. In such a situation, there appear extra nonuniversal contributions to the soft SUSY-breaking terms reflected in the combination of the nonminimality of Kälher potential and the breakdown of extra gauge symmetry. The most important one comes from the $D$-term condensations of the heavy gauge sector. This contribution is proportional to the charge of broken diagonal generators, and so we can know the large gauge symmetry by the precision measurement of scalar masses. Its phenomenological implications were discussed in Ref. [17]. Another important point is the gauge hierarchy problem. Many SUSY-GUT models achieve a small Higgs doublet masses by a fine-tuning of the parameters in the superpotential. If SUSY breaking due to the hidden field condensations is turned on, a SUSY-breaking Higgs boson mass term can become heavy and the weak scale can be destabilized. We have shown that the masses of light fields remain at the weak scale if the couplings of hidden-sector fields to visible-sector fields in the superpotential satisfy certain requirements.

We have identified the gravitino mass at the weak scale. However, our argument can be applied to models in which the gravitino mass is decoupled to the soft parameters; that is, soft parameters except gaugino masses vanish at $M$ [26]. Then we should use gaugino masses in place of $m_{3 / 2}$ as a parameter in the expansion because the scalar potential gets the radiative corrections due to gauginos.

Our procedure is applicable to effective SUGRA's derived from SST's. It is known that there are many string models with $G \times \mathrm{U}(1)^{n}[29]$ and some models have anomalous $\mathrm{U}(1)$ symmetry [30] which generates a Fayet-Iliopoulos $D$ term by one-loop effects [31]. The anomalies are canceled by the Green-Schwarz mechanism [32]. The form of the effective theory reflects the breakdown of extra gauge symmetry, and 
so it is important to search for a realistic string model by taking care of extra U(1) symmetries.

We have rederived the results in Ref. [14] by taking the limit $M_{X} / M \rightarrow 0$ and imposing some ansatz. We also have treated SUGRA with a Fayet-Iliopoulos $D$ term at the tree level independently to avoid a complication.

It is believed that measurements of the SUSY spectrum at the weak scale can be useful in probing physics at SUSY GUT, SUGRA, and/or SST, if the SUSY-breaking scenario through the gauge singlet sector in SUGRA is realized in nature. Hence precision measurements should be carried out by colliders in the near future.

\section{ACKNOWLEDGMENTS}

The author is grateful to H. Murayama, H. Nakano, and I. Joichi and especially M. Yamaguchi for useful discussions. This work was supported by the Grant-in-Aid for Scientific Research (No. 07740212) from the Japanese Ministry of Education, Science and Culture.

\section{APPENDIX A: CONSEQUENCES OF $\left\langle\partial V / \partial z^{I}\right\rangle=0$}

In this appendix, we give some consequences of the stationary conditions $\left\langle V_{I}\right\rangle \equiv\left\langle\partial V / \partial z^{I}\right\rangle=0$. From Eq. (2), we find

$$
\begin{aligned}
V_{I}= & M^{2}\left(e^{G / M^{2}}\right)_{I} U+M^{2} e^{G / M^{2}} U_{I}+\frac{1}{2}\left(\operatorname{Re} f^{-1}\right)_{\alpha \beta, I} D^{\alpha} D^{\beta} \\
& +\left(\operatorname{Re} f^{-1}\right)_{\alpha \beta} D^{\alpha}\left(D^{\beta}\right)_{I} \\
= & G_{I} e^{G / M^{2}} U+M^{2} e^{G / M^{2}}\left\{G_{I J}\left(K^{-1}\right)_{J^{\prime}}^{J} G^{J^{\prime}}\right. \\
& \left.-G_{I^{\prime}}\left(K^{-1}\right)_{J}^{I^{\prime}} K_{I J^{\prime}}^{J}\left(K^{-1}\right)_{I^{\prime \prime}}^{J^{\prime}} G^{I^{\prime \prime}}+G_{I}\right\} \\
& +\frac{1}{2}\left(\operatorname{Re} f^{-1}\right)_{\alpha \beta, I} D^{\alpha} D^{\beta}+\left(\operatorname{Re} f^{-1}\right)_{\alpha \beta} D^{\alpha}\left(z^{\dagger} T^{\beta}\right)_{J} K_{I}^{J} .
\end{aligned}
$$

Let us now multiply $\left(T^{\alpha} z\right)^{I}$ to the above, or project on a heavy-real direction. Using the identities derived from the gauge invariance of the total Kähler potential,

$$
G_{I J}\left(T^{\alpha} z\right)^{J}+G_{J}\left(T^{\alpha}\right)_{I}^{J}-K_{I}^{J}\left(z^{\dagger} T^{\alpha}\right)_{J}=0,
$$

$$
\begin{array}{r}
G_{I J J^{\prime}}\left(T^{\alpha} z\right)^{J^{\prime}}+G_{I J^{\prime}}\left(T^{\alpha}\right)_{J}^{J^{\prime}}+G_{J J^{\prime}}\left(T^{\alpha}\right)_{I}^{J^{\prime}} \\
-\left(z^{\dagger} T^{\alpha}\right)_{J^{\prime}} K_{I J}^{J^{\prime}}=0,
\end{array}
$$

$K_{I J^{\prime}}^{J}\left(T^{\alpha} z\right)^{J^{\prime}}+K_{J^{\prime}}^{J}\left(T^{\alpha}\right)_{I}^{J^{\prime}}-\left[G^{J^{\prime}}\left(z^{\dagger} T^{\alpha}\right)_{J^{\prime}}\right]_{I}^{J}=0$

we obtain

$$
\begin{aligned}
V_{I}\left(T^{\alpha} z\right)^{I}= & M^{2} e^{G / M^{2}}\left(2+U / M^{2}\right) D^{\alpha}-F^{I} F_{J}^{*}\left[G^{I^{\prime}}\left(z^{\dagger} T^{\alpha}\right)_{I^{\prime}}\right]_{I}^{J} \\
& +\frac{1}{2}\left(\operatorname{Re} f^{-1}\right)_{\beta \gamma, I}\left(T^{\alpha} z\right)^{I} D^{\beta} D^{\gamma} \\
& +\left(\operatorname{Re} f^{-1}\right)_{\beta \gamma}\left(T^{\alpha} z\right)^{I} K_{I}^{J}\left(z^{\dagger} T^{\gamma}\right)_{J} D^{\beta}
\end{aligned}
$$

Taking its VEV, we find

$$
\begin{aligned}
0= & m_{3 / 2}^{2}\left(2+\langle U\rangle / M^{2}\right)\left\langle D^{\alpha}\right\rangle-\left\langle F^{I}\right\rangle\left\langle F_{J}^{*}\right\rangle\left\langle\left[G^{I^{\prime}}\left(z^{\dagger} T^{\alpha}\right)_{I^{\prime}}\right]_{I}^{J}\right\rangle \\
& +\frac{1}{2}\left\langle\left(\operatorname{Re} f^{-1}\right)_{\beta \gamma, I}\left(T^{\alpha} z\right)^{I}\right\rangle\left\langle D^{\beta}\right\rangle\left\langle D^{\gamma}\right\rangle \\
& +\frac{1}{2}\left\langle\left(\operatorname{Re} f^{-1}\right)_{\beta \gamma}\right\rangle\left(M_{V}^{2}\right)^{\alpha \gamma}\left\langle D^{\beta}\right\rangle
\end{aligned}
$$

where $\left(M_{V}^{2}\right)^{\alpha \beta}=2\left\langle\left(z^{\dagger} T^{\beta}\right)_{J} K_{I}^{J}\left(T^{\alpha} z\right)^{I}\right\rangle$ is, up to the normalization due to the gauge coupling constants, the mass matrix of the gauge bosons. Recalling that $\left(M_{V}^{2}\right)^{\alpha \beta}$ are assumed to be $O\left(M_{X}^{2}\right)$ for broken generators of the GUT symmetry, we conclude

$$
\left\langle D^{\alpha}\right\rangle \leqslant O\left(m_{3 / 2}^{2}\right)
$$

as the first three terms of Eq. (A6) are already of order $m_{3 / 2}^{2} M_{X}^{2}$ or less. It is noteworthy that quite a similar equation to (A7) is obtained for the case of a nonlinear realization of the gauge symmetry.

From Eqs. (4) and (A7), we find $\left\langle G^{A}\right\rangle \leqslant O\left(m_{3 / 2}^{2} / M_{X}\right)$. By using relations (39), we find

$$
\begin{gathered}
\left\langle\tilde{F}_{i}^{*}\right\rangle=O\left(m_{3 / 2} M\right), \\
\left\langle F_{\kappa}^{*}\right\rangle \leqslant O\left(m_{3 / 2} M_{X}\right) .
\end{gathered}
$$

We now return to $\left\langle V_{I}\right\rangle=0$ itself. Taking the VEV of Eq. (A1) and using the relations (39) and (A7), we find

$$
\begin{gathered}
\left\langle M e^{G / 2 M^{2}} G_{I j}\right\rangle\left\langle F^{I}\right\rangle=O\left(m_{3 / 2}^{2} M\right), \\
\left\langle M e^{G / 2 M^{2}} G_{I \lambda}\right\rangle\left\langle F^{I}\right\rangle \leqslant O\left(m_{3 / 2}^{2} M_{X}\right) .
\end{gathered}
$$

Since $\left\langle M e^{G / 2 M^{2}} G_{I J}\right\rangle=\mu_{I J}+O\left(m_{3 / 2}\right)$, the above reads

$$
\begin{gathered}
\mu_{I j}\left\langle F^{I}\right\rangle=O\left(m_{3 / 2}^{2} M\right), \\
\mu_{I \lambda}\left\langle F^{I}\right\rangle \leqslant O\left(m_{3 / 2}^{2} M_{X}\right) .
\end{gathered}
$$

Since we assume that $\mu_{K L}$ is $O\left(M_{X}\right)$ for heavy complex fields, ${ }^{12}$ we find

$$
\left\langle F^{K}\right\rangle \leqslant O\left(m_{3 / 2}^{2}\right)
$$

The relation $\mu_{i j}=O\left(m_{3 / 2}\right)$ is derived from the above relations (A8) and (A12). Therefore, in our convention, the hidden-sector fields are contained in the light sector.

We can derive the following formula for the $D$-term condensation:

\footnotetext{
${ }^{12}$ Note that a careful analysis tells us that $\left\langle F^{K}\right\rangle$ $\leqslant O\left(m_{3 / 2}^{2}\left\langle z^{K}\right\rangle / M_{K}\right)$, where $M_{K}$ is the mass of $z^{K}$ from the superpotential. Thus as far as $\left\langle z^{K}\right\rangle \sim M_{K}$, the VEV of its $F$ term is always small, $\sim m_{3 / 2}^{2}$.
} 


$$
\left\langle D^{\alpha}\right\rangle=2\left(M_{V}^{-2}\right)^{\alpha \beta}\left\langle F^{I}\right\rangle\left\langle F_{J}^{*}\right\rangle\left\langle\left[G^{I^{\prime}}\left(z^{\dagger} T^{\beta}\right)_{I^{\prime}}\right]_{I}^{J}\right\rangle
$$

from Eq. (A6). We shall discuss the condition that sizable $D$-term condensations of $O\left(m_{3 / 2}^{2}\right)$ exist at $M$. In the case with a minimal Kähler potential, formula (A15) turns into a simpler form as

$$
\left\langle D^{\alpha}\right\rangle=2\left(M_{V}^{-2}\right)^{\alpha \beta}\left\langle F^{I}\right\rangle\left\langle F_{J}^{*}\right\rangle\left(T^{\beta}\right)_{I}^{J}
$$

It is shown that the $\left\langle D^{\alpha}\right\rangle$ is estimated as less than $O\left(m_{3 / 2}^{4} / M_{X}^{2}\right)$ because $\left\langle F^{K}\right\rangle \leqslant O\left(m_{3 / 2}^{2}\right)$ and $\left\langle F^{A}\right\rangle=m_{3 / 2}\left\langle G^{A}\right\rangle$ $\leqslant O\left(m_{3 / 2}^{3} / M_{X}\right)$ in the absence of a Fayet-Iliopoulos $D$ term. Hence we find that the existence of a nonminimal Kähler potential and/or Fayet-Iliopoulos $D$ term is essential to the appearance of sizable $D$-term condensations at $M$.

\section{APPENDIX B: SUGRA WITH A FAYET-ILIOPOULOS $D$ TERM}

In this appendix, we investigate the low-energy theory derived from SUGRA with a Fayet-Iliopoulos $D$ term at the tree level. This subject has not been completely examined in the literature $[33,34]$. The theory has necessarily local U(1) symmetry [27]. The superpotential is not neutral for this U(1) charge. Hereafter we denote the Fayet-Iliopoulos U(1) symmetry as $\mathrm{U}(1)_{R}$. The anomaly cancellation condition related to $\mathrm{U}(1)_{R}$ can give a strong constraint on model building [34].

Let us explain our starting point. The gauge group is $G=G_{\mathrm{SM}} \times U(1)_{R}$ where $G_{\mathrm{SM}}$ is the SM gauge group $\mathrm{SU}(3)_{C} \times \mathrm{SU}(2)_{L} \times \mathrm{U}(1)_{Y}$. Two types of chiral multiples exist. One is a set of $G_{\mathrm{SM}}$ singlet fields denoted as $\tilde{z}^{i}$. Some of them have nonzero $\mathrm{U}(1)_{R}$ charge and induce $\mathrm{U}(1)_{R}$ breaking. We assume that SUSY is broken by the $F$-term condensations of $\tilde{z}$ 's. The second one is a set of $G_{\mathrm{SM}}$ nonsinglet fields $z^{\kappa}$. For simplicity, we treat all $z^{\kappa}$ 's as light fields. Of course, we can generalize the case that the gauge group is
$G=G_{U} \times \mathrm{U}(1)_{R}$ where $G_{U}$ is a unified group.

The scalar potential is given as

$$
\begin{gathered}
V=V^{(F)}+V^{(D)} \\
V^{(F)} \equiv M^{2} \exp \left(G / M^{2}\right)\left[G^{I}\left(G^{-1}\right)_{I}^{J} G_{J}-3 M^{2}\right] \\
V^{(D)} \equiv \frac{1}{2} g_{\alpha}^{2}\left(D^{\alpha}\right)^{2}+\frac{1}{2} g_{R}^{2}\left(D^{R}\right)^{2}
\end{gathered}
$$

where the index $I, J, \ldots$ runs over all scalar species, the index $\alpha$ runs over the generators of the SM gauge group, and $D^{R} \equiv G_{I}\left(Q^{R} z\right)^{I}$. Here we denote the gauge coupling constant and $\mathrm{U}(1)$ charge of $\mathrm{U}(1)_{R}$ as $g_{R}$ and $Q_{R}$, respectively. We find that $D^{R}$ contains a constant term $Q_{R}(W) M^{2}$ since

$$
D^{R}=K_{I}\left(Q^{R} z\right)^{I}+Q_{R}(W) M^{2},
$$

where we used the fact that the superpotential carries a nonzero $U(1)_{R}$ charge $Q_{R}(W)$, i.e.,

$$
\frac{\partial W_{\mathrm{SG}}}{\partial z}\left(Q^{R} z\right)^{I}=Q_{R}(W) W_{\mathrm{SG}} .
$$

It is easy to find that a Fayet-Iliopoulos $D$ term [25] exists in the second term of $V^{(D)}$. Note that the coefficient of the Fayet-Iliopoulos $D$ term is fixed from the $\mathrm{U}(1)_{R}$ symmetry.

The $\mathrm{U}(1)_{R}$ is broken by the condensations of $\tilde{z}$ because $V^{(D)}$ is a dominant part of $V$. The orders of those VEV's are estimated as $\langle\tilde{z}\rangle=O(M)$. Hence the breaking scale of $\mathrm{U}(1)_{R}$ is of order $M$.

Now we compute the scalar potential of the low-energy effective theory by taking the flat limit and integrating out the heavy fields in $\tilde{z}$ 's simultaneously. The $D$-term contribution is added to the scalar masses in comparison with the result in Ref. [21]. We write it down in the form that the scalar masses are read off,

$$
\begin{gathered}
V^{(\mathrm{FI})}=V_{\mathrm{SUSY}}^{(\mathrm{FI})}+V_{\mathrm{soft}}^{(\mathrm{FI})}+\Delta V^{(\mathrm{FI})}, \\
V_{\mathrm{SUSY}}^{(\mathrm{FI})}=\left|\frac{\partial \hat{\mathscr{W}}}{\partial z^{\kappa}}\right|^{2}+\frac{1}{2} g_{\alpha}^{2}\left[z_{\kappa}^{*}\left(T^{\alpha}\right)_{\lambda}^{\kappa} z^{\lambda}\right]^{2}, \\
V_{\mathrm{soft}}^{(\mathrm{FI})}=A \hat{W}+B^{\kappa}(z) \frac{\partial \hat{\mathscr{W}}}{\partial z^{\kappa}}+\text { H.c. }+\left\{\left.|| m_{3 / 2}\right|^{2}+\frac{\langle V\rangle}{M^{2}}\right) \delta_{\kappa}^{\lambda}+\left\langle\tilde{F}^{i}\right\rangle\left(K_{i \kappa}^{\mu} K_{\mu}^{j \lambda}-K_{i \kappa}^{j \lambda}\right)\left\langle\tilde{F}_{j}^{*}\right\rangle \\
\left.+g_{R}^{2}\left\langle D^{R}\right\rangle Q_{\kappa}^{R} \delta_{\kappa}^{\lambda}\right\} z^{\kappa} z_{\lambda}^{*}+\left\{-\left\langle\tilde{F}^{i}\right\rangle H_{i \kappa \lambda}^{j}\left\langle\tilde{F}_{j}^{*}\right\rangle+m_{3 / 2}\left\langle\tilde{F}^{i}\right\rangle H_{i \kappa \lambda}+m_{3 / 2}^{*}\left\langle\tilde{F}_{j}^{*}\right\rangle H_{\kappa \lambda}^{j}\right\} z^{\kappa} z^{\lambda}+\text { H.c. }, \\
\Delta V^{(\mathrm{FI})}=\frac{\partial \hat{W}^{*}}{\partial \tilde{z}_{i}^{*}}\left\langle\left(K^{-1}\right)_{i}^{j}\right\rangle \frac{\partial \hat{W}}{\partial \tilde{z}^{j}}+\left\langle\tilde{F}^{i}\right\rangle \frac{\partial \hat{W}}{\partial \tilde{z}^{i}}+\text { H.c. },
\end{gathered}
$$

up to constant terms and higher order terms of $O\left(m_{3 / 2}^{5} / M\right)$. Here $B^{\kappa}(z), C\left(z, z^{*}\right), \hat{\mathscr{W}}$, and $\hat{W}$ have been already defined in Sec. II C.

We find that the $\mathrm{U}(1)_{R} D$-term contribution to scalar masses can destroy universality among scalar masses at $M$. (Its existence was suggested in Ref. [33], but we have proved it by deriving the full low-energy scalar potential from SUGRA directly.) As its contribution is proportional to the $\mathrm{U}(1)_{R}$ charge, the $\mathrm{U}(1)_{R}$ charge of matters can be known from measurements of the weak scale SUSY spectrum. 
[1] M.J.G. Veltman, Acta Phys. Pol. B 12, 437 (1981); L. Maiani, Gif-sur-Yvette Summer School on Particle Physics, 11th, Gifsur-Yvette, France, 1979 (Inst. Nat. Phys. Nucl. Phys. Particules, Paris, 1979); E. Witten, Nucl. Phys. B185, 513 (1981); M. Dine, W. Fischler, and M. Srednicki, ibid. B189, 575 (1981); S. Dimopoulos and S. Raby, ibid. B192, 353 (1981).

[2] JLC Group, JLC-I:KEK Report No. 92-16, 1992 (unpublished).

[3] E. Cremmer, B. Julia, J. Scherk, S. Ferrara, L. Girardello, and P. van Nieuwenhuizen, Phys. Lett. 79B, 231 (1978); Nucl. Phys. B147, 105 (1979); E. Cremmer, S. Ferrara, L. Girardello, and A. van Proeyen, Phys. Lett. 116B, 231 (1982); Nucl. Phys. B212, 413 (1983).

[4] A.H. Chamseddine, R. Arnowitt, and P. Nath, Phys. Rev. Lett. 49, 970 (1982); R. Barbieri, S. Ferrara, and C.A. Savoy, Phys. Lett. 119B, 343 (1982).

[5] G.L. Kane, C. Kolda, L. Roszkowski, and J.D. Wells, Phys. Rev. D 49, 6173 (1994), and references therein.

[6] J. Ellis and D.V. Nanopoulos, Phys. Lett. 110B, 44 (1982); R. Barbieri and R. Gatto, ibid. 110B, 211 (1982); T. Inami and C.S. Lim, Nucl. Phys. B207, 533 (1982).

[7] L.E. Ibáñez and D. Lüst, Nucl. Phys. B382, 305 (1992); B. de Carlos, J.A. Casas, and C. Muñoz, Phys. Lett. B 299, 234 (1993); V.S. Kaplunovsky and J. Louis, ibid. 306, 269 (1993).

[8] N.V. Krasnikov, A.B. Kyiatkin, and E.R. Poppitz, Phys. Lett. B 222, 66 (1989); Sov. J. Nucl. Phys. 50, 901 (1989); M.K. Gaillard and V. Jain, Phys. Rev. D 49, 1951 (1994).

[9] S. Dimopoulos and H. Georgi, Nucl. Phys. B193, 150 (1981); N. Sakai, Z. Phys. C 11, 153 (1981).

[10] H. Georgi and S.L. Glashow, Phys. Rev. Lett. 32, 438 (1974).

[11] LEP Collaborations, Phys. Lett. B 276, 247 (1992); P. Langacker and M.-X. Luo, Phys. Rev. D 44, 817 (1991); U. Amaldi, W. de Boer, and H. Fürstenau, Phys. Lett. B 260, 447 (1991); W.J. Marciano, Annu. Rev. Nucl. Part. Sci. 41, 469 (1991).

[12] P. Nath and R. Arnowitt, Phys. Rev. D 38, 1479 (1988); J. Hisano, H. Murayama, and T. Yanagida, Nucl. Phys. B402, 46 (1993).

[13] B. Gato, J. León, J.P. Mercader, and M. Quiros, Nucl. Phys. B253, 285 (1985); P. Moxhay and K. Yamamoto, ibid. B256, 130 (1985); K. Grassie, Phys. Lett. 159B, 32 (1985); B. Gato, Nucl. Phys. B278, 189 (1986).

[14] Y. Kawamura, H. Murayama, and M. Yamaguchi, Phys. Rev. D 51, 1337 (1995).

[15] R. Hempfling, Phys. Rev. D 52, 4106 (1995); L.J. Hall, R. Rattazzi, and U. Sarid, Report No. SU-ITP-94/15, RU-94-37 1994 (unpublished); N. Polonsky and A. Pomarol, Phys. Rev. Lett. 73, 2295 (1994); D. Matalliotakis and H.P. Nilles, Nucl.
Phys. B435, 115 (1995); D. Olechowski and S. Pokorski, Phys. Lett. B 344, 201 (1995).

[16] T. Tsukamoto, K. Fujii, H. Murayama, M. Yamaguchi, and Y. Okada, Phys. Rev. D 51, 3153 (1995).

[17] Y. Kawamura, H. Murayama, and M. Yamaguchi, Phys. Lett. B 324, 54 (1994); Y. Kawamura and M. Tanaka, Prog. Theor. Phys. 91, 949 (1994); 93, 789 (1995).

[18] L. Hall, J. Lykken, and S. Weinberg, Phys. Rev. D 27, 2359 (1983).

[19] M. Drees, Phys. Rev. D 33, 1468 (1986).

[20] K. Inoue, A. Kakuto, H. Komatsu, and S. Takeshita, Prog. Theor. Phys. 68, 927 (1982); 71, 413 (1984); L.E. Ibáñez, Phys. Lett. 118B, 73 (1982); Nucl. Phys. B218, 514 (1983); L. Alvarez-Gaumé, J. Polchinski, and M. Wise, ibid. B221, 495 (1983); J. Ellis, J.S. Hagelin, D.V. Nanopoulos, and K. Tamvakis, Phys. Lett. 125B, 275 (1983).

[21] S.K. Soni and H.A. Weldon, Phys. Lett. 126B, 215 (1983).

[22] G.F. Giudice and A. Masiero, Phys. Lett. B 206, 148 (1988).

[23] M. Drees, Phys. Lett. B 181, 279 (1986); J.S. Hagelin and S. Kelley, Nucl. Phys. B342, 95 (1990).

[24] A.E. Faraggi, J.S. Hagelin, S. Kelley, and D.V. Nanopoulos, Phys. Rev. D 45, 3272 (1992).

[25] P. Fayet and J. Iliopoulos, Phys. Lett. 51B, 416 (1974); P. Fayet, Nucl. Phys. B90, 104 (1975).

[26] J. Ellis, C. Kounnas, and D.V. Nanopoulos, Phys. Lett. 143B, 410 (1984); J. Ellis, K. Enqvist, and D.V. Nanopoulos, ibid. 147B, 99 (1984).

[27] R. Barbieri, S. Ferrara, D.V. Nanopoulos, and K.S. Stelle, Phys. Lett. 113B, 219 (1982); S. Ferrara, L. Girardello, T. Kugo, and A. Van Proeyen, Nucl. Phys. B223, 191 (1983).

[28] I. Joichi, Y. Kawamura, and M. Yamaguchi, Touhoku Report No. TU-459, DPSU-9403 (unpublished).

[29] L.E. Ibáñez, J.E. Kim, H.P. Nilles, and F. Quevedo, Phys. Lett. B 191, 282 (1987); A. Font, L.E. Ibáñez, F. Quevedo, and A. Sierra, Nucl. Phys. B331, 421 (1990); Y. Katsuki, Y. Kawamura, T. Kobayashi, N. Ohtsubo, Y. Ono, and K. Tanioka, ibid. B341, 611 (1990).

[30] J.A. Casas and C. Muñoz, Phys. Lett. B 209, 214 (1988); 212, 343 (1988); 214, 63 (1988).

[31] M. Dine, N. Seiberg, and E. Witten, Nucl. Phys. B289, 589 (1987).

[32] M.B. Green and J.H. Schwarz, Phys. Lett. 149B, 117 (1984).

[33] H. Nakano, Report No. KUNS 1257, HE(TH)94/05, 1994 (unpublished).

[34] Minimal SUGRA with a Fayet-Iliopoulos $D$ term has been examined in A.H. Chamseddine and H. Dreiner, Nucl. Phys. B458, 65 (1995). 